\title{
The ionization fraction gradient across the Horsehead edge: an archetype for molecular clouds ${ }^{\star}$
}

\author{
J. R. Goicoechea ${ }^{1}$, J. Pety ${ }^{2,3}$, M. Gerin ${ }^{3}$, P. Hily-Blant ${ }^{4}$, and J. Le Bourlot ${ }^{5}$ \\ ${ }^{1}$ Laboratorio de Astrofísica Molecular. Centro de Astrobiología. CSIC-INTA. Carretera de Ajalvir, Km 4. Torrejón de Ardoz, \\ 28850 Madrid, Spain \\ e-mail: goicoechea@damir.iem.csic.es \\ 2 IRAM, 300 rue de la Piscine, 38406 Grenoble Cedex, France \\ e-mail: pety@iram.fr \\ 3 LERMA - LRA, UMR 8112, CNRS, Observatoire de Paris and École Normale Supérieure, 24 rue Lhomond, 75231 Paris, France \\ e-mail: maryvonne.gerin@lra.ens.fr \\ ${ }^{4}$ Laboratoire d'Astrophysique, Observatoire de Grenoble, BP 53, 38041 Grenoble Cedex 09, France \\ e-mail: pierre.hilyblant@obs.ujf-grenoble.fr \\ 5 LUTH, UMR 8102 CNRS, Université Paris 7 and Observatoire de Paris, Place J. Janssen, 92195 Meudon, France \\ e-mail: Jacques.Lebourlot@obspm.fr
}

Received 10 December 2008 / Accepted 11 February 2009

\begin{abstract}
Context. The ionization fraction (i.e., the electron abundance) plays a key role in the chemistry and dynamics of molecular clouds. Aims. We study the $\mathrm{H}^{13} \mathrm{CO}^{+}, \mathrm{DCO}^{+}$and $\mathrm{HOC}^{+}$line emission towards the Horsehead, from the shielded core to the UV irradiated cloud edge, i.e., the photodissociation region (PDR), as a template to investigate the ionization fraction gradient in molecular clouds. Methods. We analyze an IRAM Plateau de Bure Interferometer map of the $\mathrm{H}^{13} \mathrm{CO}^{+} J=1-0$ line at a $6.8^{\prime \prime} \times 4.7^{\prime \prime}$ resolution, complemented with IRAM-30 $\mathrm{m} \mathrm{H}^{13} \mathrm{CO}^{+}$and $\mathrm{DCO}^{+}$higher- $J$ line maps and new $\mathrm{HOC}^{+}$and $\mathrm{CO}^{+}$observations. We compare self-consistently the observed spatial distribution and line intensities with detailed depth-dependent predictions of a PDR model coupled with a nonlocal radiative transfer calculation. The chemical network includes deuterated species, ${ }^{13} \mathrm{C}$ fractionation reactions and $\mathrm{HCO}^{+} / \mathrm{HOC}^{+}$ isomerization reactions. The role of neutral and charged PAHs in the cloud chemistry and ionization balance is investigated.

Results. The detection of the $\mathrm{HOC}^{+}$reactive ion towards the Horsehead PDR proves the high ionization fraction of the outer UV irradiated regions, where we derive a low $\left[\mathrm{HCO}^{+}\right] /\left[\mathrm{HOC}^{+}\right] \simeq 75-200$ abundance ratio. In the absence of PAHs, we reproduce the observations with gas-phase metal abundances, $[\mathrm{Fe}+\mathrm{Mg}+\ldots]$, lower than $4 \times 10^{-9}$ (with respect to $\mathrm{H}$ ), and a cosmic-ray ionization rate of $\zeta=(5 \pm 3) \times 10^{-17} \mathrm{~s}^{-1}$. The inclusion of PAHs modifies the ionization fraction gradient and increases the required metal abundance. Conclusions. The ionization fraction in the Horsehead edge follows a steep gradient, with a scale length of $\sim 0.05 \mathrm{pc}$ (or $\sim 25^{\prime \prime}$ ), from $\left[\mathrm{e}^{-}\right] \simeq 10^{-4}$ (or $n_{\mathrm{e}} \sim 1-5 \mathrm{~cm}^{-3}$ ) in the PDR to a few times $\sim 10^{-9}$ in the core. $\mathrm{PAH}^{-}$anions play a role in the charge balance of the cold and neutral gas if substantial amounts of free PAHs are present $\left([\mathrm{PAH}]>10^{-8}\right)$.
\end{abstract}

Key words. astrochemistry - ISM: clouds - radiative transfer - radio lines: ISM - ISM: molecules - ISM: abundances

\section{Introduction}

The electron abundance $\left(\left[\mathrm{e}^{-}\right]=n_{\mathrm{e}} / n_{\mathrm{H}}\right)$ plays a fundamental role in the chemistry and dynamics of interstellar gas. The degree of ionization determines the preponderance of ionneutral reactions, i.e., the main formation route for most chemical species in molecular clouds (Herbst \& Klemperer 1973; Oppenheimer \& Dalgarno 1974). In addition, the ionization fraction constrains the coupling of matter and magnetic fields, which drives the dissipation of turbulence and the transfer of angular momentum, thus having crucial implications in protostellar collapse and accretion disks (e.g., Balbus \& Hawley 1991).

High-angular resolution observations of interstellar clouds reveal steep density, temperature and turbulence gradients as well as sharp chemical variations. Accordingly, the electron

* Based on observations obtained with the IRAM Plateau de Bure interferometer and $30 \mathrm{~m}$ telescope. IRAM is supported by INSU/CNRS (France), MPG (Germany), and IGN (Spain). abundance should vary within a cloud depending on the relative ionizing sources and prevailing chemistry.

Rotational line emission of molecular ions such as $\mathrm{DCO}^{+}$ and $\mathrm{HCO}^{+}$have been traditionally used to estimate the ionization fraction in molecular clouds because (i) they are abundant and easily observable; (ii) dissociative recombination is their main destruction route, and thus their abundances are roughly inversely proportional to the electron abundance (e.g., Guélin et al. 1982; Wootten et al. 1982; de Boisanger et al. 1996; Williams et al. 1998; Caselli et al. 1998; Maret \& Bergin 2007; Hezareh et al. 2008). On the other hand, the presence of reactive ions (species such as $\mathrm{HOC}^{+}$or $\mathrm{CO}^{+}$that react rapidly with $\mathrm{H}_{2}$ ) is predicted to be a sensitive indicator of high ionization fraction regions, e.g., the UV irradiated cloud surfaces (e.g., Smith et al. 2002; Fuente et al. 2003).

In order to constrain the ionization fraction gradient from models, the cloud chemistry and physics cannot be simplified much because the charge balance depends on parameters such 
Table 1. Observation parameters of the PdBI maps shown in Fig. 1.

\begin{tabular}{|c|c|c|c|c|c|c|c|c|c|c|c|}
\hline Molecule & Transition & $\begin{array}{r}\text { Frequency } \\
\mathrm{GHz}\end{array}$ & Instrument & Config. & $\begin{array}{l}\text { Beam } \\
\text { arcsec }\end{array}$ & $\begin{array}{c}\text { PA } \\
\circ\end{array}$ & $\begin{array}{c}\text { Vel. Resol. } \\
\mathrm{km} \mathrm{s}^{-1}\end{array}$ & $\begin{array}{c}\text { Int. Time }^{a} \\
\text { hours }\end{array}$ & $\begin{array}{c}T_{\text {sys }} \\
\mathrm{K}\end{array}$ & $\begin{array}{c}\text { Noise }^{b, \dagger} \\
\text { K }\end{array}$ & Obs. date \\
\hline $\mathrm{H}^{13} \mathrm{CO}^{+}$ & $1-0$ & 86.754288 & PdBI & $C \& D$ & $6.8 \times 4.7$ & 13 & 0.2 & 6.5 & 150 & 0.10 & 2006-07 \\
\hline $\mathrm{HCO}$ & $1_{0,1} 3 / 2,2-0_{0,0} 1 / 2,1$ & 86.670760 & PdBI & $C \& D$ & $6.7 \times 4.4$ & 16 & 0.2 & 6.5 & 150 & 0.09 & 2006-07 \\
\hline
\end{tabular}

${ }^{a}$ We observed a 7-field mosaic centered on the IR peak at $\alpha_{2000}=05^{\mathrm{h}} 40^{\mathrm{m}} 54.27^{\mathrm{s}}, \delta_{2000}=-02^{\circ} 28^{\prime} 00^{\prime \prime}$ (Abergel et al. 2003) with the following offsets: $\left(-5.5^{\prime \prime},-22.0^{\prime \prime}\right),\left(5.5^{\prime \prime},-22.0^{\prime \prime}\right),\left(11.0^{\prime \prime}, 0.0^{\prime \prime}\right),\left(0.0^{\prime \prime}, 0.0^{\prime \prime}\right),\left(-11.0^{\prime \prime}, 0.0^{\prime \prime}\right),\left(-5.5^{\prime \prime}, 22.0^{\prime \prime}\right)$ and $\left(5.5^{\prime \prime}, 22.0^{\prime \prime}\right)$. The total field-of-view is $80.1^{\prime \prime} \times 102.1^{\prime \prime}$ and the half power primary beam is 58.1". The mosaic was Nyquist sampled in declination at 3.4 mm and largely oversampled in right ascension. This maximizes the field of view along the PDR edge while the oversampling in the perpendicular direction eases the deconvolution. On-source time was computed as if the source was always observed with 6 antennae; ${ }^{b}$ the noise values refer to the mosaic phase center (mosaic noise is inhomogeneous due to primary beam correction; it steeply increases at the mosaic edges).

Table 2. Observation parameters of the IRAM-30 m observations.

\begin{tabular}{|c|c|c|c|c|c|c|c|c|c|c|c|}
\hline Molecule & Transition & $\begin{array}{r}\text { Frequency } \\
\mathrm{GHz}\end{array}$ & Instrument & $F_{\text {eff }}$ & $B_{\text {eff }}$ & $\begin{array}{l}\text { Resol. } \\
\text { arcsec }\end{array}$ & $\begin{array}{l}\text { Resol. } \\
\mathrm{km} \mathrm{s}^{-1}\end{array}$ & $\begin{array}{l}\text { Int. Time } \\
\text { hours }\end{array}$ & $\begin{array}{c}\text { Noise }^{\dagger} \\
\text { K }\end{array}$ & $\begin{array}{l}\text { Observing } \\
\text { Mode }\end{array}$ & Obs. date \\
\hline $\mathrm{HCO}^{+}$ & $J=1-0$ & 89.188523 & $30 \mathrm{~m} / \mathrm{A} 100$ & 0.95 & 0.78 & $27.6^{\prime \prime}$ & 0.20 & 4.7 & 0.02 & ON-OFF & 2008 \\
\hline $\mathrm{HOC}^{+}$ & $J=1-0$ & 89.487414 & $30 \mathrm{~m} / \mathrm{A} 100$ & 0.95 & 0.78 & $27.5^{\prime \prime}$ & 0.20 & 4.7 & 0.02 & ON-OFF & 2008 \\
\hline $\mathrm{CO}^{+}$ & $2,5 / 2-1,3 / 2$ & 236.062578 & $30 \mathrm{~m} / \mathrm{A} 230$ & 0.91 & 0.52 & $10.4^{\prime \prime}$ & 0.20 & 4.7 & 0.05 & ON-OFF & 2008 \\
\hline $\mathrm{H}^{13} \mathrm{CO}^{+}$ & $J=1-0$ & 86.754288 & $30 \mathrm{~m} / \mathrm{AB} 100$ & 0.95 & 0.78 & $28.4^{\prime \prime}$ & 0.20 & 2.6 & 0.10 & OTF map & $2006-07$ \\
\hline $\mathrm{H}^{13} \mathrm{CO}^{+}$ & $J=3-2$ & 260.255339 & $30 \mathrm{~m} /$ HERA & 0.90 & 0.46 & $13.5^{\prime \prime}$ & 0.20 & 5.9 & 0.06 & OTF map & 2006 \\
\hline $\mathrm{DCO}^{+}$ & $J=2-1$ & 144.077289 & $30 \mathrm{~m} / \mathrm{CD} 150$ & 0.93 & 0.69 & $18.0^{\prime \prime}$ & 0.08 & 5.9 & 0.18 & OTF map & 2006 \\
\hline $\mathrm{DCO}^{+}$ & $J=3-2$ & 216.112582 & $30 \mathrm{~m} / \mathrm{HERA}$ & 0.90 & 0.52 & $11.4^{\prime \prime}$ & 0.11 & 1.5 & 0.10 & OTF map & 2006 \\
\hline
\end{tabular}

$\dagger$ The noise (in $T_{\mathrm{mb}}$ scale) refers to the channel spacing obtained by averaging adjacent channels to the velocity resolution given in the tables.

as the penetration of UV radiation, the cosmic-ray ionization rate $(\zeta)$ and the abundance of key species (e.g., metals and PAH).

Compared to other works, in this paper we determine the ionization fraction gradient by direct comparison of $\mathrm{H}^{13} \mathrm{CO}^{+}$ and $\mathrm{DCO}^{+}$high-angular resolution maps and $\mathrm{HOC}^{+}$pointed observations, with detailed depth-dependent chemical and radiative transfer models covering a broad range of cloud physical conditions. Indeed, the observed field-of-view contains the famous Horsehead PDR (the UV illuminated edge of the cloud) and a dense and cold core discovered by us from its intense $\mathrm{DCO}^{+}$line emission (Pety et al. 2007). Due to its simple geometry and moderate distance $(d \simeq 400 \mathrm{pc})$, the Horsehead PDR and associated core are good templates to study the steep gradients expected in molecular clouds (e.g., Pety et al. 2005, 2007; Goicoechea et al. 2006; Gerin et al. 2009).

The paper is organized as follows. The observations are presented in Sect. 2 and the models used to interpret them are described in Sect. 3. The chemistry of $\mathrm{H}^{13} \mathrm{CO}^{+}, \mathrm{DCO}^{+}$and $\mathrm{HOC}^{+}$ (our observational probes of the ionization fraction) is analyzed in Sect. 4. In Sect. 5 we investigate the role of metals, PAHs and $\zeta$ on the electron abundance determination. The main results and constrains are presented in Sect. 6 and discussed in Sect. 7.

\section{Observations}

\subsection{Observations and data reduction}

Tables 1 and 2 summarize the observation parameters of the data obtained with the PdBI and the IRAM-30 m telescope that we shall study in this work. The $\mathrm{H}^{13} \mathrm{CO}^{+} J=1-0$ line emission map was first presented in Gerin et al. (2009). Frequency-switched, on-the-fly maps (OTF) obtained at the IRAM- $30 \mathrm{~m}$ were used to produce the short-spacings needed to complement a 7-field mosaic acquired with the $6 \mathrm{PdBI}$ antennae in the $\mathrm{CD}$ configuration (baseline lengths from 24 to $176 \mathrm{~m}$ ). Correlator backends were used (VESPA for IRAM-30 m observations). The high angular resolution $\mathrm{PdBI} \mathrm{H}^{13} \mathrm{CO}^{+} J=1-0$ map complements our previous $\mathrm{H}^{13} \mathrm{CO}^{+} J=3-2$ and $\mathrm{DCO}^{+} J=2-1$ and 3-2 maps taken with the IRAM-30 m telescope and first presented in Pety et al. (2007).

In this work we present new IRAM-30 $\mathrm{m}$ deeper integrations in the $\mathrm{HOC}^{+}, \mathrm{H}^{13} \mathrm{CO}^{+}$and $\mathrm{HCO}^{+} J=1-0$ lines, and an upper limit for the $\mathrm{CO}^{+}$emission towards the PDR (defined here as the HCO emission peak; Gerin et al. 2009). The position switching observing mode was used. The on-off cycle duration was $1 \mathrm{mn}$ and the off-position offsets were $(\Delta \alpha, \Delta \delta)=\left(-100^{\prime \prime}, 0^{\prime \prime}\right)$, i.e., the $\mathrm{H}$ II region ionized by $\sigma$ Ori and free of molecular gas emission. Position accuracy is estimated to be $\sim 3^{\prime \prime}$ for the $30 \mathrm{~m}$ data and better than $0.5^{\prime \prime}$ for the PdBI data. The data processing was done with the GILDAS ${ }^{1}$ softwares (e.g., Pety 2005b). The IRAM-30 m data were first calibrated to the $T_{\mathrm{A}}^{*}$ scale using the chopper wheel method (Penzias \& Burrus 1973), and finally converted to main beam temperatures $T_{\mathrm{mb}}$ using the forward and main beam efficiencies $F_{\text {eff }}$ and $B_{\text {eff }}$ displayed in Table 2 (e.g., Greve et al. 1998). The amplitude accuracy for heterodyne observations with the IRAM-30 m telescope is $\sim 10 \%$. PdBI data and short-spacing data were merged before imaging and deconvolution of the mosaic, using standard techniques of GILDAS and used in our previous works (see e.g., Pety et al. 2005).

\section{2. $\mathrm{DCO}^{+}$and $\mathrm{H}^{13} \mathrm{CO}^{+}$spatial distribution, $\mathrm{HOC}^{+}$detection}

Figure 1 shows $\mathrm{H}^{13} \mathrm{CO}^{+} J=1-0, \mathrm{HCO} 1_{0,1}-0_{0,0}(\mathrm{PdBI})$ and $\mathrm{DCO}^{+} J=2-1,3-2$ integrated line intensity maps (IRAM-30 m; Pety et al. 2007), as well as the aromatic infrared band emission (AIB, observed with ISOCAM, Abergel et al. 2003) that traces the UV illuminated edge of the cloud, i.e., the PDR. The $\mathrm{DCO}^{+}$emission is concentrated in a narrow, arclike structure of dense and cold gas behind the PDR (Pety et al. 2007). Hence, it shows a very different spatial distribution than the emission of "PDR tracers" such as $\mathrm{C}_{2} \mathrm{H}, \mathrm{C}_{4} \mathrm{H}, c-\mathrm{C}_{3} \mathrm{H}_{2}$ (Pety et al. 2005), HCO radicals (Gerin et al. 2009), vibrationally excited $\mathrm{H}_{2}$ (Habart et al. 2005) or the AIB emission (Compiègne et al. 2008). The $\mathrm{H}^{13} \mathrm{CO}^{+} J=1-0$ emission follows the $\mathrm{DCO}^{+}$

\footnotetext{
${ }^{1}$ See http://www.iram. fr/IRAMFR/GILDAS
} 

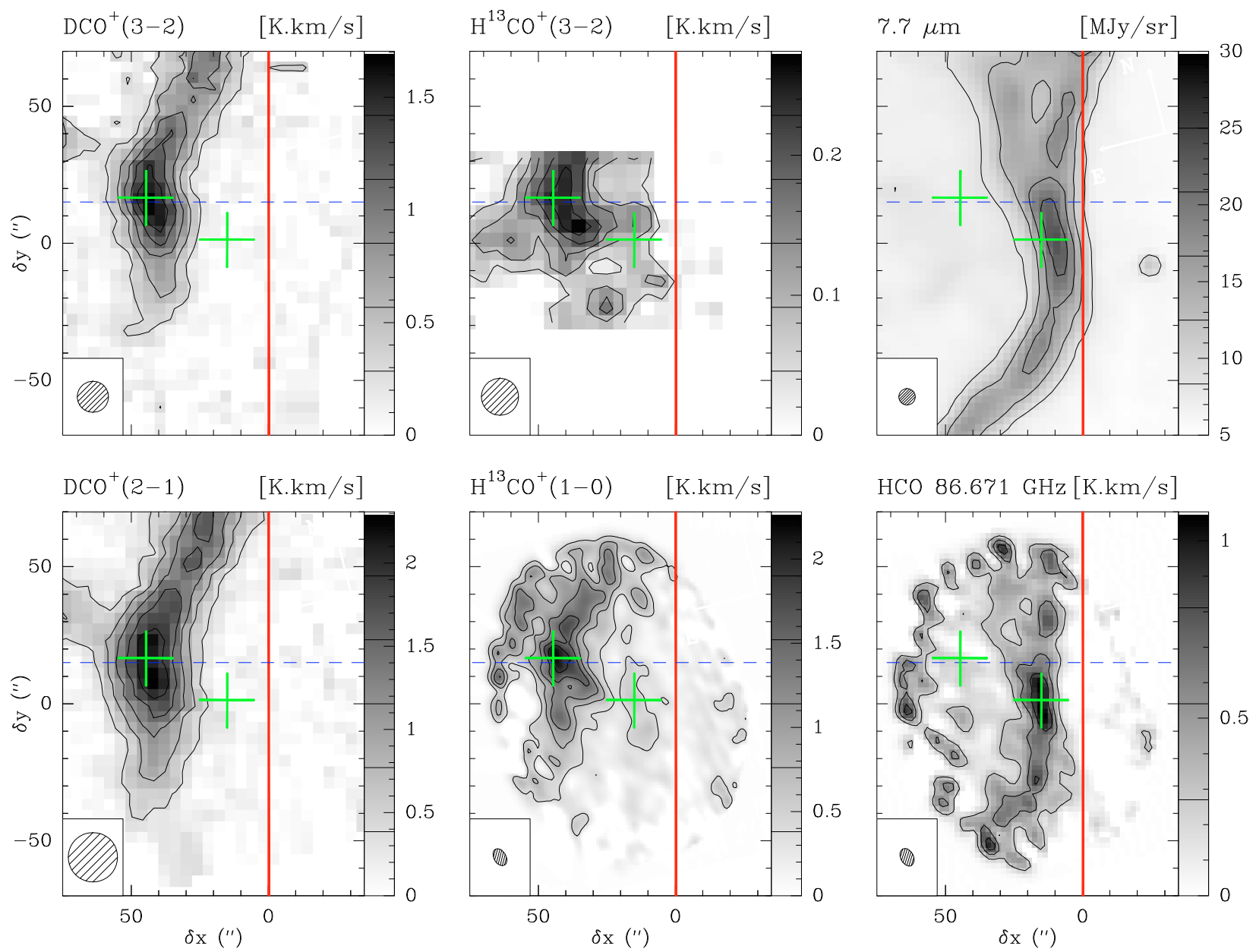

Fig. 1. $\mathrm{DCO}^{+} J=3-2$ and 2-1 (IRAM-30 m; from Pety et al. 2007), $\mathrm{H}^{13} \mathrm{CO}^{+} J=1-0$ (PdBI) and 3-2 (IRAM-30 m) line integrated intensity maps, aromatic infrared band emission (ISOCAM, from Abergel et al. 2003) and HCO (PdBI, from Gerin et al. 2009). Maps have been rotated by $14^{\circ}$ counter-clockwise around the projection center, located at $(\delta x, \delta y)=\left(20^{\prime \prime}, 0^{\prime \prime}\right)$, to bring the illuminated star direction in the horizontal direction. The horizontal zero has been set at the cloud edge $\left(\delta x=0^{\prime \prime}\right)$. The $\mathrm{H}^{13} \mathrm{CO}^{+}, \mathrm{DCO}^{+}$and $\mathrm{HCO}$ emission is integrated between 10.1 and $11.1 \mathrm{~km} \mathrm{~s}^{-1}$. Integrated intensities are expressed in the $T_{\mathrm{mb}}$ scale. Contour levels are displayed on the grey scale lookup tables. The red vertical line shows the PDR edge and the green crosses shows two representative positions: the "shielded core" (the $\mathrm{DCO}^{+}$emission peak at $\delta x \sim 45^{\prime}$; Pety et al. 2007) and the "PDR" (the HCO emission peak at $\delta x \sim 15^{\prime}$; Gerin et al. 2009). The dashed blue line shows the horizontal cut analyzed in this work.

distribution and it mostly delineates the dense core that coincides with the $\mathrm{DCO}^{+}$emission peak. Nevertheless, while $\mathrm{DCO}^{+}$ is not detected in the illuminated edge, $\mathrm{H}^{13} \mathrm{CO}^{+}$does show a faint emission in the PDR. Therefore, the small field-of-view shown in Fig. 1 contains two different environments: a warm PDR and a cold core shielded from the external UV radiation field. In the following sections we analyze these emission maps to determine the ionization fraction gradient in the region.

Figure 2 shows long integration spectra of the $\mathrm{HOC}^{+}$, $\mathrm{H}^{13} \mathrm{CO}^{+}$and $\mathrm{HCO}^{+} J=1-0$ lines towards the PDR. This is the first detection of the $\mathrm{HOC}^{+}$reactive ion towards the Horsehead, and adds to previous detections in interstellar environments with high electron abundances (Woods et al. 1983; Ziurys \& Apponi 1995; Fuente et al. 2003; Rizzo et al. 2003; Savage \& Ziurys 2004; Liszt et al. 2004). $\mathrm{H}^{12} \mathrm{CO}^{+}$lines are optically thick, as shown by the low $\mathrm{H}^{12} \mathrm{CO}^{+} / \mathrm{H}^{13} \mathrm{CO}^{+} J=1-0$ line intensity ratio $(\sim 7)$, much lower than the expected ${ }^{12} \mathrm{C} /{ }^{13} \mathrm{C} \simeq$ 60 abundance ratio (Langer \& Penzias 1990; Savage et al. 2002) and references therein). The high opacity of $\mathrm{H}^{12} \mathrm{CO}^{+}$lines even towards the PDR justifies the use of $\mathrm{H}^{13} \mathrm{CO}^{+}$lines as tracers of the $\mathrm{HCO}^{+}$abundance.
Table 3. Main spectroscopic parameters of the studied lines.

\begin{tabular}{lcccc}
\hline \hline Species & $\begin{array}{c}\text { Transition } \\
J_{\text {upp }}-J_{\text {low }}\end{array}$ & $\begin{array}{c}\text { Frequency } \\
(\mathrm{GHz})\end{array}$ & $\begin{array}{c}A_{\mathrm{ij}} \\
\left(\mathrm{s}^{-1}\right)\end{array}$ & $\begin{array}{c}E_{\text {upp }} \\
(\mathrm{K})\end{array}$ \\
\hline $\mathrm{HCO}^{+}$ & $1-0$ & 89.188523 & $4.2 \times 10^{-5}$ & 4.3 \\
$\mathrm{HOC}^{+}$ & $1-0$ & 89.487414 & $2.2 \times 10^{-5}$ & 4.3 \\
$\mathrm{H}^{13} \mathrm{CO}^{+}$ & $1-0$ & 86.754288 & $3.9 \times 10^{-5}$ & 4.2 \\
$\mathrm{CO}^{+}$ & $2(5 / 2)-1(3 / 2)$ & 236.062578 & $4.7 \times 10^{-4}$ & 17.2 \\
$\mathrm{H}^{13} \mathrm{CO}^{+}$ & $3-2$ & 260.255339 & $1.3 \times 10^{-3}$ & 25.0 \\
$\mathrm{DCO}^{+}$ & $2-1$ & 144.077289 & $2.1 \times 10^{-4}$ & 10.4 \\
$\mathrm{DCO}^{+}$ & $3-2$ & 216.112582 & $7.7 \times 10^{-4}$ & 20.7 \\
\hline
\end{tabular}

\section{Analysis: models}

In this work we couple the depth-dependent abundances predicted by a PDR model (for the varying physical conditions prevailing in the Horsehead edge) with detailed excitation and radiative transfer calculations adapted to the cloud geometry. This technique allows us to analyze different chemical models by direct comparison with observed line intensities. This methodology was introduced to study our interferometric CS and $\mathrm{C}^{18} \mathrm{O}$ 


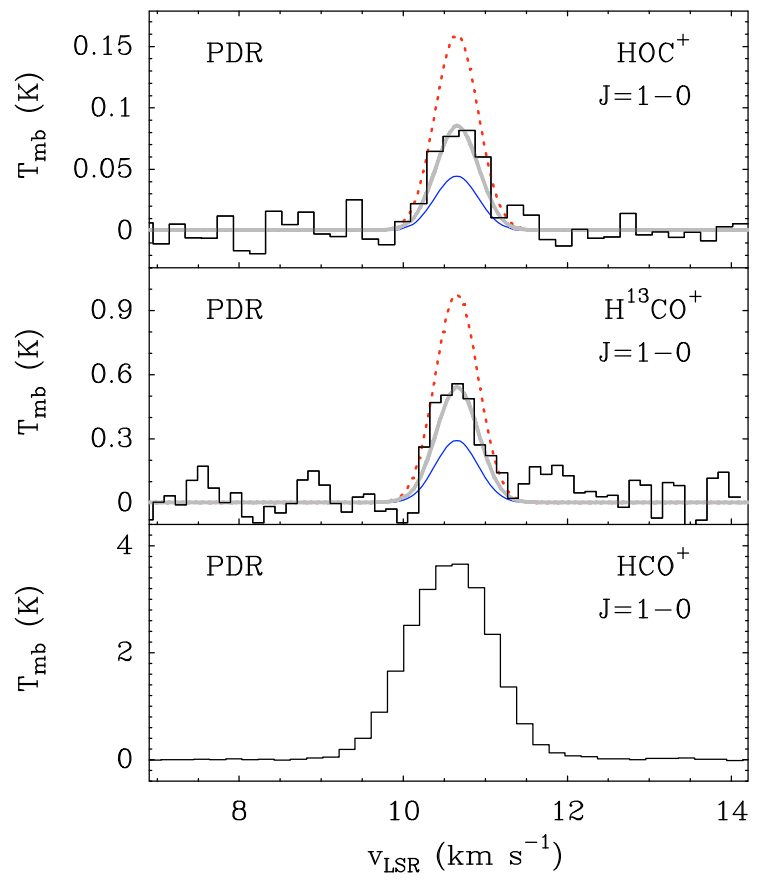

Fig. 2. $\mathrm{HOC}^{+}$and $\mathrm{H}^{13} \mathrm{CO}^{+} J=1-0$ lines towards the Horsehead PDR (upper and middle panels) observed with the IRAM-30 $\mathrm{m}$ telescope. Solid lines are radiative transfer models with $T_{\mathrm{k}}=60 \mathrm{~K}, n\left(\mathrm{H}_{2}\right)=$ $5 \times 10^{4} \mathrm{~cm}^{-3}, n(\mathrm{H})=500 \mathrm{~cm}^{-3}$ and $\left[\mathrm{e}^{-}\right]=5 \times 10^{-5}$. Three different abundances are shown, thick-grey line: $\left[\mathrm{HOC}^{+}\right]=4.0 \times 10^{-12}$ and $\left[\mathrm{H}^{13} \mathrm{CO}^{+}\right]=1.5 \times 10^{-11}$; red dashed line: abundances $\times 2$; blue thin line: abundances $\div 2$. For completeness, the $\mathrm{HCO}^{+} J=1-0$ line towards the PDR is also shown (lower panel). This transition is very opaque, as shown by the low $\mathrm{H}^{12} \mathrm{CO}^{+} / \mathrm{H}^{13} \mathrm{CO}^{+} J=1-0$ line intensity ratio $(\sim 7)$. The resulting line profile is thus broadened and it suffers from scattering by low-density foreground gas that we do not model here.

maps of the Horsehead edge (Goicoechea et al. 2006). It enables us to observationally benchmark the abundance gradients predicted by chemical models, even if it does not produce perfect fits to line profiles in all cloud positions. In this paper, we analyze a horizontal cut of the $\mathrm{H}^{13} \mathrm{CO}^{+}$and $\mathrm{DCO}^{+}$line emission along the direction of the illuminating star $\left(\delta y=15^{\prime \prime}\right)$. Figure 1 shows that this cut (blue dashed line) goes across the $\mathrm{DCO}^{+}$emission peak $\left(\delta x \sim 45^{\prime \prime}\right)$, which we identify as the "shielded core", and across the HCO emission peak, the "PDR" $\left(\delta x \sim 15^{\prime \prime}\right)$.

\subsection{Geometry and density gradient}

The Horsehead edge has an almost edge-on geometry with a line-of-sight depth of $l_{\text {depth }} \simeq 0.1$ pc (e.g., Habart et al. 2005) and a spatial scale in the plane of the sky of $\simeq 0.002 \mathrm{pc} \mathrm{arcsec}^{-1}$. We determine the density profile from observations by fitting the $1.2 \mathrm{~mm}$ dust continuum emission (IRAM-30 m/MAMBO) along the $\delta y=15^{\prime \prime}$ direction (Hily-Blant et al. 2005). In this fit, we adopt a dust opacity per unit (gas+dust) mass column density of $\kappa_{1.2}=0.003 \mathrm{~cm}^{2} \mathrm{~g}^{-1}$ at $1.2 \mathrm{~mm}$ (computed for "MRN grains": Mathis et al. 1977, see below), our best knowledge of the dust grains temperature (from $\sim 15 \mathrm{~K}$ in the core to $\sim 30 \mathrm{~K}$ in the PDR; e.g., Ward-Thompson et al. 2006) and a power-law density profile $n_{\mathrm{H}}(r)=n(\mathrm{H})+2 n\left(\mathrm{H}_{2}\right) \propto r^{-p}$, where $r$ is the distance from the shielded core towards the illuminated edge of the cloud. Best fits are obtained for a steep density gradient in the cloud edge $(p \simeq 3)$ and a flatter one towards the core $(p \simeq 0.5)$. The turnover point occurs at a core radius of $r \simeq 0.04 \mathrm{pc}$ (or $\delta x \simeq 23^{\prime \prime}$ in the maps). The resulting density gradient used in the photochemical and radiative transfer models is shown in Fig. 4. In the next sections we constrain the ionization fraction gradient in the cloud by comparing synthetic and observed $\mathrm{H}^{13} \mathrm{CO}^{+}$and $\mathrm{DCO}^{+}$spectra along the same cut.

\subsection{Photochemical models}

We have updated the Meudon PDR code to model our observations of the Horsehead. The code has been described in detail elsewhere (e.g., Le Bourlot et al. 1993; Le Petit et al. 2006; Goicoechea \& Le Bourlot 2007) and benchmarked against other PDR codes by Röllig et al. (2007). In this section we summarize the most relevant upgrades and model features for this work.

\subsubsection{UV radiative transfer and dust properties}

The code solves the UV radiative transfer problem taking into account dust scattering and gas absorption. Anisotropic scattering of UV photons by dust grains is included by explicity calculating the wavelength-dependent grain albedo and $g$-asymmetry parameters (Goicoechea \& Le Bourlot 2007). This enables the specific computation of the UV radiation field (continuum+lines) and thus, the direct integration of consistent photoionization and photodissociation rates. We use two types of dust populations: (i) a mixture of graphite+silicate grains; and (ii) PAHs (see next paragraph). More precisely, we adopt a power-law size distribution $\left(n(a) \propto a^{-3.5}\right)$ with minimum and maximum radius of $\sim 5$ and $\sim 250 \mathrm{~nm}$ respectively (for graphite+silicate grains). Wavelength-dependent optical properties ( $Q$ efficiencies and $g$ factors) are interpolated from Laor $\&$ Draine (1993) tabulations. With a standard gas-to-dust mass ratio $(\sim 100)$, this grain mixture ("MRN grains") reproduces the main characteristics of the standard interstellar extinction curve with $N_{\mathrm{H}} / A_{\mathrm{V}}=1.9 \times 10^{21} \mathrm{~cm}^{-2}$ and $R_{V}=3.1$.

In order to complete our description of the dust populations, in this work we have also added smaller aromatic grains. Observationally, the AIB emission towards the Horsehead (produced by free PAHs according to the most accepted theory; Léger \& Puget 1984; Allamandola et al. 1985) clearly separates the H II region and PDR (where the emission is bright) from the regions shielded from UV radiation, where no AIB emission is detected (Abergel et al. 2003; Habart et al. 2005; Compiègne et al. 2007; 2008). However, the size distribution and PAH abundance in dense regions shielded from UV radiation are uncertain. It may vary from "negligible", if PAHs coagulate into larger PAH aggregates (Boulanger et al. 1990; Rapacioli et al. 2006) to "high" abundances (though they will not be detected in the mid-IR due to the lack of UV photons to excite them). We used the following PAH properties: a size distribution with $\sim 0.4$ and $\sim 1.2 \mathrm{~nm}$ radii limits (Desert et al. 1990) and optical parameters from $\mathrm{Li}$ \& Draine (2001). This size distribution is compatible with PAHs having a mean radius of $\sim 0.6 \mathrm{~nm}$ and $N_{\mathrm{C}} \sim 100$ carbon atoms assuming $N_{\mathrm{C}} \simeq 500 a^{3}$ (Bakes \& Tielens 1994). The extinction curve and the efficiency of the photoelectric heating mechanism depend on the mass fraction put into PAHs (Bakes \& Tielens 1994). Depending on the PAH abundance, they contribute to the total dust mass by $\sim 1 \%$ for $[\mathrm{PAH}]=10^{-7}$ and $\sim 10 \%$ for $[\mathrm{PAH}]=10^{-6}$. 
Table 4. Key chemical reaction rates ${ }^{\dagger}$ adopted in this work.

\begin{tabular}{ll}
\hline \hline Reaction & Rate $\left[\mathrm{cm}^{3} \mathrm{~s}^{-1}\right]$ \\
\hline $\mathrm{HCO}^{+}+\mathrm{e}^{-} \rightarrow \mathrm{CO}+\mathrm{H}$ & $2.4 \times 10^{-7}(300 \mathrm{~K} / \mathrm{T})^{0.69 a}$ \\
$\mathrm{HCO}^{+}+\mathrm{PAH} \rightarrow \mathrm{PAH}+\mathrm{CO}+\mathrm{H}$ & $1.4 \times 10^{-8}(300 \mathrm{~K} / \mathrm{T})^{0.50 b}$ \\
$\mathrm{M}^{+}+\mathrm{e}^{-} \rightarrow \mathrm{M}+\mathrm{h} v$ & $3.7 \times 10^{-12}(300 \mathrm{~K} / \mathrm{T})^{0.65}$ \\
$\mathrm{M}^{+}+\mathrm{PAH}^{-} \rightarrow \mathrm{M}+\mathrm{PAH}$ & $1.0 \times 10^{-8}(300 \mathrm{~K} / \mathrm{T})^{0.50 b}$ \\
$\mathrm{C}^{+}+\mathrm{H}_{2} \mathrm{O} \rightarrow \mathrm{HCO}^{+}+\mathrm{H}$ & $8.9 \times 10^{-10}(300 \mathrm{~K} / \mathrm{T})^{0.50}$ \\
$\mathrm{C}^{+}+\mathrm{H}_{2} \mathrm{O} \rightarrow \mathrm{HOC}^{+}+\mathrm{H}$ & $1.8 \times 10^{-9}(300 \mathrm{~K} / \mathrm{T})^{0.50}$ \\
$\mathrm{CO}^{+}+\mathrm{H}_{2} \rightarrow \mathrm{HCO}^{+}+\mathrm{H}$ & $7.5 \times 10^{-10}$ \\
$\mathrm{CO}^{+}+\mathrm{H}_{2} \rightarrow \mathrm{HOC}^{+}+\mathrm{H}$ & $7.5 \times 10^{-10}$ \\
$\mathrm{HOC}^{+}+\mathrm{H}_{2} \rightarrow \mathrm{HCO}^{+}+\mathrm{H}_{2}$ & $3.8 \times 10^{-10 c}$ \\
\hline
\end{tabular}

Rates are from the UDFA and $O S U$ databases unless indicated; ${ }^{a}$ cited in the text as $\alpha\left(\mathrm{HCO}^{+}\right)$, it also applies to $\mathrm{DCO}^{+}$and $\mathrm{H}^{13} \mathrm{CO}^{+}$; ${ }^{b}$ rate is from (Flower \& Pineau des Forêts 2003); ${ }^{c}$ rate is from (Smith et al. 2002).

\subsubsection{Chemical network and elemental abundances}

Once the UV field is determined at each cloud position, steady-state chemical abundances are computed for a given network of chemical reactions. The model also computes the temperature profile by solving the thermal balance between the most important gas heating and cooling mechanisms (Le Petit et al. 2006). Our chemical network contains $\sim 160$ species and $\sim 2000$ reactions. It includes deuteration, ${ }^{13} \mathrm{C}$ fractionation (Graedel et al. 1982) and $\mathrm{HCO}^{+} / \mathrm{HOC}^{+}$isomerization reactions. When available, we used the photodissociation rates given by van Dishoeck (1988), which are explicitly calculated for Draine's interstellar radiation field (ISRF). The most critical reaction rates for our determination of the ionization fraction are listed in Table 4. Most reactions were checked against $O S U$ (Herbst and co-workers) and UDFA (Woodall et al. 2006) networks. Also, we benchmarked our network with more extended ones by comparing the predicted abundances of simple species such as $\mathrm{CO}$ and $\mathrm{DCO}^{+}$.

Following Flower \& Pineau des Forêts (2003), we have also included interactions ( $\sim 60$ reactions) of gas phase species with very small aromatic grains (neutral PAH and singly charged $\mathrm{PAH}^{ \pm}$). In particular, we take into account PAHgas processes such as neutralization reactions of atomic and molecular cations on $\mathrm{PAH}^{-}$, $\mathrm{PAH}$ electron attachment and photodetachment of $\mathrm{PAH}^{-}$and $\mathrm{PAH}$ by UV photons. Such processes can play a significant role in the ionization balance of dense molecular clouds (e.g., Lepp \& Dalgarno 1988; Bakes \& Tielens 1998; Flower et al. 2007, Wakelam \& Herbst 2008; Wolfire et al. 2008). We have not included larger grains in the network in order to isolate the role of PAHs in the gasphase chemistry. We thus assume that recombinations of ions with grains are much less frequent than recombinations with electrons and $\mathrm{PAH}^{-}$. This is partially justified by the fact that, according to their size and mass, the fractional abundance of "MRN grains" is low: $n_{\mathrm{g}} / n_{\mathrm{H}} \approx 10^{-10}$ and their effective cross section per $\mathrm{H}$ nucleus is $\left(n_{\mathrm{g}} / n_{\mathrm{H}}\right) \pi a^{2} \approx 10^{-21} \mathrm{~cm}^{-2}$ (the product of the grain abundance and the mean grain cross section). Grain growth towards the denser parts the cloud will result in even lower grain abundances and smaller effective cross sections if the gas-to-dust mass ratio has to be preserved: e.g., $\left(n_{\mathrm{g}} / n_{\mathrm{H}}\right) \approx 10^{-13}$ and $\left(n_{\mathrm{g}} / n_{\mathrm{H}}\right) \pi a^{2} \approx 10^{-22} \mathrm{~cm}^{-2}$ if the grain radii $a$ increase by $\sim 10$. Therefore, the resulting lower abundance of charged grains and their smaller effective cross section for ion-grain recombinations will not alter our estimations of the ionization fraction much.
Table 5. Standard conditions and gas-phase elemental abundances. Molecular, atomic and electron abundances, noted $[\mathrm{x}]$, refer to $\mathrm{H}$.

\begin{tabular}{lc}
\hline \hline Parameter & Value \\
\hline Radiation field $\chi$ & 60 (Draine units) \\
Density $n_{\mathrm{H}}(r)=n(\mathrm{H})+2 n\left(\mathrm{H}_{2}\right)$ & $\propto r^{-3}$, up to $\sim 2 \times 10^{5} \mathrm{~cm}^{-3}$ \\
Line of sight depth $l_{\text {depth }}$ & $0.1 \mathrm{pc}$ \\
{$[\mathrm{He}]=n(\mathrm{He}) / n_{\mathrm{H}}$} & $1.00 \times 10^{-1}$ \\
{$[\mathrm{O}]$} & $3.02 \times 10^{-4}$ \\
{$\left[{ }^{12} \mathrm{C}\right]$} & $1.38 \times 10^{-4}$ \\
{$[\mathrm{~N}]$} & $7.95 \times 10^{-5}$ \\
{$[\mathrm{D}]$} & $1.60 \times 10^{-5}$ \\
{$[\mathrm{~S}]$} & $3.50 \times 10^{-6}$ \\
{$\left[{ }^{13} \mathrm{C}\right]=\left[{ }^{12} \mathrm{C}\right] / 60$} & $2.30 \times 10^{-6}$ \\
{$[\mathrm{PAH}]$} & variable: $0-10^{-7}$ \\
{$[\mathrm{metals}] \equiv[\mathrm{M}] \equiv[\mathrm{Fe}+\mathrm{Mg}+\ldots]$} & variable: $10^{-11}-10^{-5}$ \\
Cosmic-ray ionization rate $\zeta$ & variable: $10^{-18}-10^{-15} \mathrm{~s}^{-1}$ \\
\hline
\end{tabular}

The adopted elemental abundances are shown in Table 5. Low ionization potential heavy metals $\left(\lesssim 8 \mathrm{eV} ; \mathrm{Fe}^{+}, \mathrm{Mg}^{+}\right.$or $\left.\mathrm{Na}^{+}\right)$ are all represented by a single element, " $\mathrm{M}^{+}$". In our model, such metals slowly recombine with electrons (through radiative recombinations), can be photoionized and can exchange charge with ions and neutrals (including PAHs). However, they are assumed to be chemically inert and thus do not form metallic molecules (e.g., Oppenheimer \& Dalgarno 1974).

Once the physical and geometrical parameters of the cloud are constrained, the only free parameters in the model are the cosmic-ray ionization rate and the metal and PAH abundances.

\subsection{From abundances to spectra: $\mathrm{mm}$ radiative transfer}

We use the PDR model predictions (molecular abundance, $n\left(\mathrm{H}_{2}\right), n(\mathrm{H})$, gas temperature and ionization fraction gradients) as input for a nonlocal radiative transfer calculation able to compute $\mathrm{DCO}^{+}$and $\mathrm{H}^{13} \mathrm{CO}^{+}$line intensities as a function of cloud position. Our radiative transfer code handles edge-on planeparallel geometry, and accounts for line trapping, collisional excitation $^{2}$, and radiative excitation by absorption of continuum photons. After the level populations are determined in each modeled slab, emergent line intensities along each line of sight are computed and convolved with the telescope angular resolution at each frequency. A more detailed description is given in Goicoechea et al. (2006; Appendix). Since typical densities in the Horsehead $\left(\sim 10^{4}-10^{5} \mathrm{~cm}^{-3}\right)$ are below the critical densities of the observed high-dipole moment molecular ions (a few $\sim 10^{5}-10^{6} \mathrm{~cm}^{-3}$ for the studied transitions) our approach allows us to properly take into account non-LTE excitation effects (e.g., subthermal excitation), as well as opacity and line profile formation.

\section{Chemistry of the ionization fraction probes}

\section{1. $\mathrm{H}^{13} \mathrm{CO}^{+}$and $\mathrm{DCO}^{+}$chemistry in the UV shielded core}

The detection of very bright $\mathrm{DCO}^{+}$emission towards the shielded core (Pety et al. 2007) implies cold gas temperatures $\left(T_{\mathrm{k}} \simeq 10-20 \mathrm{~K}\right)$ and thus efficient $\mathrm{HCO}^{+}$deuterium fractionation (i.e., $\left[\mathrm{DCO}^{+}\right] /\left[\mathrm{HCO}^{+}\right] \gg \mathrm{D} / \mathrm{H}$ ). From our observations we

2 PDR-like environments require us to consider inelastic collisions with $\mathrm{H}_{2}, \mathrm{H}, \mathrm{He}$ and $\mathrm{e}^{-} . \mathrm{H}^{13} \mathrm{CO}^{+}, \mathrm{DCO}^{+}$and $\mathrm{HOC}^{+}$collisional rates with $\mathrm{H}_{2}, \mathrm{H}$ and $\mathrm{He}$ have been scaled from those of Flower (1999), while collisional rates with $\mathrm{e}^{-}$were kindly provided by Faure and Tennyson (see e.g., Faure \& Tennyson 2001). 
infer $\left[\mathrm{DCO}^{+}\right] \simeq 8.0 \times 10^{-11},\left[\mathrm{H}^{13} \mathrm{CO}^{+}\right] \simeq 6.5 \times 10^{-11}$ and thus a $\left[\mathrm{DCO}^{+}\right] /\left[\mathrm{HCO}^{+}\right] \simeq 0.02$ abundance ratio towards the core peak. Such gas-phase $\mathrm{DCO}^{+}$enhancement is achieved via the reaction:

$\mathrm{H}_{3}^{+}+\mathrm{HD} \rightleftarrows \mathrm{H}_{2} \mathrm{D}^{+}+\mathrm{H}_{2}+\Delta \mathrm{E}$

which is endothermic by $\sim 232 \mathrm{~K}$ in the right-to-left direction (Gerlich et al. 2002), followed by

$\mathrm{CO}+\mathrm{H}_{2} \mathrm{D}^{+} \rightarrow \mathrm{DCO}^{+}+\mathrm{H}_{2}$

which dominates the $\mathrm{DCO}^{+}$formation in the cold and dense gas. The absence of significant $\mathrm{DCO}^{+}$line emission in the PDR is consistent with the higher temperatures $(>60 \mathrm{~K})$ expected in the illuminated edge of the cloud.

The detection of intense $\mathrm{H}^{13} \mathrm{CO}^{+}$emission towards the shielded core and its vicinity (see Fig. 1) implies low ionization fractions. In terms of excitation and opacity effects, $\mathrm{H}^{13} \mathrm{CO}^{+}$is a much more reliable tracer of $\mathrm{HCO}^{+}$column density than $\mathrm{H}^{12} \mathrm{CO}^{+}$itself (as the latter suffers from very large opacities and line photon scattering by low-density halos; e.g., Cernicharo \& Guélin 1987). In terms of its chemistry, two main processes dominate the formation of $\mathrm{H}^{13} \mathrm{CO}^{+}$in the low temperature shielded gas:

${ }^{13} \mathrm{CO}+\mathrm{H}_{3}^{+} \rightarrow \mathrm{H}^{13} \mathrm{CO}^{+}+\mathrm{H}_{2}$

and isotopic fractionation through

${ }^{13} \mathrm{CO}+\mathrm{H}^{12} \mathrm{CO}^{+} \rightleftarrows \mathrm{H}^{13} \mathrm{CO}^{+}+{ }^{12} \mathrm{CO}+\Delta \mathrm{E}$

which is endothermic by only $\sim 9 \mathrm{~K}$ in the right-to-left direction (Langer et al. 1984) and competes with dissociative recombination in the destruction of $\mathrm{H}^{13} \mathrm{CO}^{+}$where the abundance of electrons is low. For the physical conditions prevailing in the shielded core, we predict $\left[\mathrm{H}^{12} \mathrm{CO}^{+}\right] /\left[\mathrm{H}^{13} \mathrm{CO}^{+}\right]$abundance ratios down to $\sim 0.7$ times lower than the elemental $\left[{ }^{12} \mathrm{C}\right] /\left[{ }^{13} \mathrm{C}\right]$ isotopic ratio. Since both $\mathrm{H}^{13} \mathrm{CO}^{+}$and $\mathrm{DCO}^{+}$are mainly destroyed by fast dissociative recombination with electrons:

$\mathrm{H}^{13} \mathrm{CO}^{+}+\mathrm{e}^{-} \rightarrow{ }^{13} \mathrm{CO}+\mathrm{H}$

$\mathrm{DCO}^{+}+\mathrm{e}^{-} \rightarrow \mathrm{CO}+\mathrm{D}$

their abundances inversely scale with that of electrons. In this work we have used a "standard" $\mathrm{HCO}^{+}$dissociative recombination rate (i.e., $\alpha\left(\mathrm{HCO}^{+}\right)=2.4 \times 10^{-7}(300 / T)^{0.69} \mathrm{~cm}^{3} \mathrm{~s}^{-1}$ ) recommended in most astrochemical databases. We note, however, that there is a certain discrepancy among different theoretical calculations and measurements of this key chemical rate (see discussion by Florescu-Mitchell \& Mitchell 2006, and references therein). In Sect. 7 we discuss the influence of adopting a smaller, "non standard" $\alpha^{\prime}\left(\mathrm{HCO}^{+}\right)$rate on our results.

\section{2. $\mathrm{HOC}^{+}$and $\mathrm{H}^{13} \mathrm{CO}^{+}$chemistry in the PDR}

In order to extract the $\left[\mathrm{HOC}^{+}\right]$and $\left[\mathrm{H}^{13} \mathrm{CO}^{+}\right]$abundances towards the Horsehead PDR, we have modeled the observed lines (Fig. 2) using our best knowledge of the prevailing physical conditions: $T_{\mathrm{k}}=60-120 \mathrm{~K}, n\left(\mathrm{H}_{2}\right)=5 \times 10^{4} \mathrm{~cm}^{-3}$, $n(\mathrm{H})=500 \mathrm{~cm}^{-3},\left[\mathrm{e}^{-}\right]=5 \times 10^{-5}$ and a $0.1 \mathrm{pc}$ line-of-sight depth (or $N_{\mathrm{H}} \simeq 3.1 \times 10^{22} \mathrm{~cm}^{-2}$ ) all accurate within a factor $\sim 2$. From the observed lines we derive the following column densities: $N\left(\mathrm{HOC}^{+}\right)=(1.2-2.5) \times 10^{11} \mathrm{~cm}^{-2}$ and $N\left(\mathrm{H}^{13} \mathrm{CO}^{+}\right)=(4.7-$ $7.8) \times 10^{11} \mathrm{~cm}^{-2}$, which translates into $\left[\mathrm{HOC}^{+}\right]=(0.4-0.8) \times$ $10^{-11}$ and $\left[\mathrm{H}^{13} \mathrm{CO}^{+}\right]=(1.5-2.5) \times 10^{-11}$. This computation assumes that the $\mathrm{HOC}^{+}$and $\mathrm{H}^{13} \mathrm{CO}^{+}$emission fills the IRAM$30 \mathrm{~m}$ beam. However, $\mathrm{HOC}^{+}$has not been mapped and its

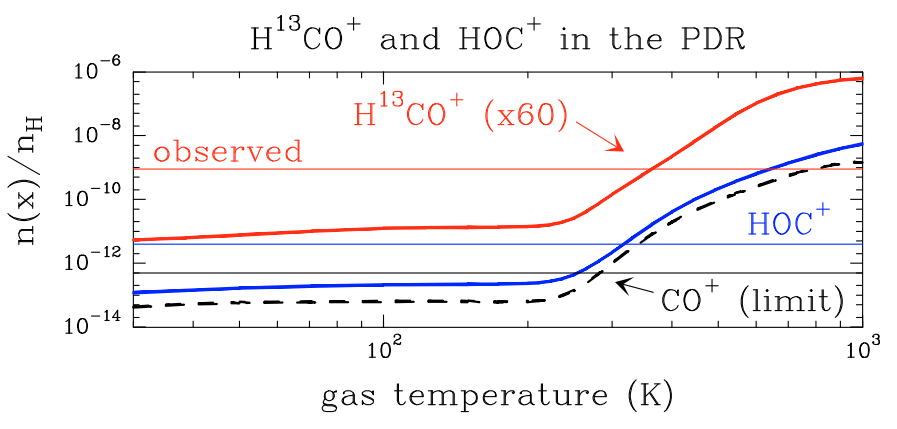

Fig. 3. Predicted $\mathrm{H}^{13} \mathrm{CO}^{+}, \mathrm{HOC}^{+}$and $\mathrm{CO}^{+}$peak abundances in the PDR $\left(A_{\mathrm{V}} \simeq 0.5-1.5\right)$ as a function of gas temperature. $\mathrm{H}^{13} \mathrm{CO}^{+}$and $\mathrm{HOC}^{+}$ abundances (and $\mathrm{CO}^{+}$abundance upper limit) derived from observations towards the PDR position are shown with horizontal thin lines.

emission could well arise from the same $\sim 12^{\prime \prime}$-width filament where the emission of small hydrocarbons and $\mathrm{HCO}$ radical is concentrated (Pety et al. 2005; Gerin et al. 2009). In this case, $\left[\mathrm{HOC}^{+}\right]$increases by a factor $\sim 3$. Therefore, we conclude that the $\left[\mathrm{HOC}^{+}\right] /\left[\mathrm{H}^{13} \mathrm{CO}^{+}\right]$abundance ratio towards the PDR lies in the range $\simeq 0.3-0.8$. These values are orders of magnitude higher than the value expected in the UV shielded gas.

Our chemical models (see next section) reproduce the $\left[\mathrm{HOC}^{+}\right] /\left[\mathrm{H}^{13} \mathrm{CO}^{+}\right]$abundance ratio towards the PDR but the absolute abundances derived from observations are larger than those predicted by the model. The discrepancies between observed and modeled abundances for $\mathrm{HOC}^{+}$and $\mathrm{H}^{13} \mathrm{CO}^{+}$likely have a common origin. In particular, the formation of $\mathrm{HOC}^{+}$in $\mathrm{UV}$ irradiated gas is driven by reactions involving $\mathrm{C}^{+}$and species such as $\mathrm{H}_{2} \mathrm{O}$ and $\mathrm{CO}^{+}$(from $\mathrm{C}^{+}+\mathrm{OH}$ reaction) that efficiently form at high temperatures, that is:

$$
\begin{aligned}
& \mathrm{C}^{+}+\mathrm{H}_{2} \mathrm{O} \rightarrow \mathrm{HCO}^{+} / \mathrm{HOC}^{+}+\mathrm{H} \\
& \mathrm{CO}^{+}+\mathrm{H}_{2} \rightarrow \mathrm{HCO}^{+} / \mathrm{HOC}^{+}+\mathrm{H}
\end{aligned}
$$

where reaction 7 predominantly produces $\mathrm{HOC}^{+}$whereas reaction 8 has similar branching ratios for the $\mathrm{HCO}^{+}$and $\mathrm{HOC}^{+}$ formation (e.g., Scott et al. 1997; Savage \& Ziurys 2004). The $\mathrm{HOC}^{+}$destruction is governed by the isomerization reaction:

$\mathrm{HOC}^{+}+\mathrm{H}_{2} \rightarrow \mathrm{HCO}^{+}+\mathrm{H}_{2}$.

Laboratory experiments show that the reaction rate is lower than previously thought (Smith et al. 2002), allowing interstellar $\mathrm{HOC}^{+}$to exist at detectable amounts.

The intensity peak of the $\mathrm{CO} J=2-1$ optically thick lines observed with the $\operatorname{PdBI}\left(T_{\mathrm{mb}} \simeq 60 \mathrm{~K} \approx T_{\mathrm{ex}}\right.$; Pety et al. 2005), together with the observed $\mathrm{CO} J=4-3 / 2-1$ line ratio (Philipp et al. 2006), provide a lower limit to the gas temperature in the PDR $\left(T_{\mathrm{k}} \simeq 60-120 \mathrm{~K}\right)$. Temperatures in this range are predicted by the PDR model but are not enough to overcome the activation energy barriers of the neutralneutral reactions leading to the formation of abundant $\mathrm{H}_{2} \mathrm{O}$,

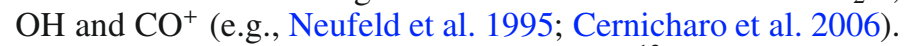
Therefore, our models predict $\mathrm{HOC}^{+}$and $\mathrm{H}^{13} \mathrm{CO}^{+}$abundances lower than observed because their precursor molecules have low abundances, and reactions 7 and 8 are not efficient enough.

We have computed that gas temperatures around $\sim 350 \mathrm{~K}$ are needed to reproduce the observed $\mathrm{HOC}^{+}$and $\mathrm{H}^{13} \mathrm{CO}^{+}$abundances in the PDR through the previous scheme (see Fig. 3). Our models of the Horsehead (low UV radiation field) include photoelectric heating from PAHs and grains but do not predict such a warm gas component even if the PAH abundance is significantly 
increased. However, we do not model the PDR gas dynamics and thus processes such as shock waves, driven by the expansion of the $\mathrm{H}$ II region that compress the cloud edge, may provide additional gas heating sources to trigger this warm chemistry. This reasoning is partially consistent with the non detection of $\mathrm{CO}^{+}$ lines, at least at the sensitivity level of our long integration observation $\left(\mathrm{rms} \sim 50 \mathrm{mK}\right.$ in a $0.20 \mathrm{~km} \mathrm{~s}^{-1}$ velocity width channel or $\left.\left[\mathrm{CO}^{+}\right] \leq 5 \times 10^{-13}\right)$.

If the gas in the PDR has not gone through such a warm phase, reaction 8 has to be ruled out as the main chemical pathway for $\mathrm{HOC}^{+}$formation and an alternative formation scenario is required. In this case, we propose that the enhanced $\mathrm{HOC}^{+}$ abundance in the PDR can still be related to the high abundance of $\mathrm{C}^{+}$(and thus high ionization fraction), but also to grain photodesorption of water-ice mantles formed in earlier evolutionary stages of the cloud.

In this picture, the low $\chi / n$ ratio in the Horsehead $\left(\sim 10^{-3}\right)$ will allow water-ices to be photodesorbed close to the illuminated edge of the cloud (see predictions by Hollenbach et al. 2009), increasing the water vapor abundance well above the pure gas phase predictions. Reaction 7 will then dominate the $\mathrm{HOC}^{+}$formation in the PDR. Taking into account that isomerization, dissociative recombination and photodissociation contribute to $\mathrm{HOC}^{+}$destruction, we estimate that the required water vapor abundance needed to explain the inferred $\mathrm{HOC}^{+}$abundance in the PDR is $\left[\mathrm{H}_{2} \mathrm{O}\right] \simeq 1800 \times\left[\mathrm{HOC}^{+}\right] \simeq$ $(0.7-2.2) \times 10^{-8}$. Herschel Space Observatory observations will enable the detection of $\mathrm{C}^{+}$and $\mathrm{H}_{2} \mathrm{O}$ lines in a large sample of PDRs, confirming whether or not water vapor is abundant at the edges of molecular clouds (e.g., Cernicharo et al. 2006).

\section{Determination of the ionization fraction}

Figure 4 presents depth-dependent predictions of several photochemical models across the Horsehead edge. Each model shows the main physical parameters (density and temperature), the ionization fraction gradient, the $\mathrm{DCO}^{+}, \mathrm{H}^{13} \mathrm{CO}^{+}$and $\mathrm{HOC}^{+}$abundances (our observational probes of the ionization fraction) and the abundances of key chemical species for the charge balance in the cloud: $\mathrm{CO} / \mathrm{C} / \mathrm{C}^{+}, \mathrm{M} / \mathrm{M}^{+}, \mathrm{PAH}^{-} / \mathrm{PAH} / \mathrm{PAH}^{+}$.

Four sets of models are displayed. Top/bottom models use a low $\left(\zeta=3 \times 10^{-17} \mathrm{~s}^{-1}\right)$ and high $\left(\zeta=3 \times 10^{-16} \mathrm{~s}^{-1}\right)$ cosmicray ionization rate respectively. Left/right models exclude and include the effects of PAHs. In the latter case, we include PAHs in the UV radiative transfer (as a source of absorption and scattering of UV photons), in the photoelectric heating and in the chemical network. We start the chemistry computation by including neutral PAHs alone with an initial abundance of $[\mathrm{PAH}]=10^{-7}$. In each set of models (each panel), the only parameter that varies is the abundance of metals: high metallicity with $[\mathrm{M}]=10^{-6}$ (dashed curves) and low metallicity with $[\mathrm{M}]=10^{-9}$ (solid curves). The low metallicity case implies a large metal depletion from the gas phase.

In terms of the chemical species observed in this work, a salient feature of all models is the constancy of the $\mathrm{DCO}^{+} / \mathrm{H}^{13} \mathrm{CO}^{+}$abundance ratio once the gas is shielded from $\mathrm{UV}$ radiation $\left(A_{\mathrm{V}} \gtrsim 6\right)$. This feature agrees with the almost identical spatial distribution of $\mathrm{DCO}^{+}$and $\mathrm{H}^{13} \mathrm{CO}^{+}$emission observed beyond the PDR (see Fig. 1). This similarity was already noticed in the lower resolution $\mathrm{DCO}^{+}$and $\mathrm{H}^{13} \mathrm{CO}^{+}$pioneering maps of several molecular clouds (e.g., Guélin et al. 1982). Also, the predicted $\left[\mathrm{HOC}^{+}\right] /\left[\mathrm{H}^{13} \mathrm{CO}^{+}\right]$abundance ratio towards the $\mathrm{PDR}$ is in good agreement with the value inferred from observations. In this UV irradiated region where the $\mathrm{C}^{+}$and electron abundances are very high, the $\mathrm{HCO}^{+}$destruction rate becomes comparable to the isomerization rate (reaction 9). This implies that the $\left[\mathrm{HOC}^{+}\right] /\left[\mathrm{H}^{13} \mathrm{CO}^{+}\right]$abundance ratio in the cloud achieves the highest value in the PDR.

\subsection{The role of ionized carbon and metals}

According to the ionization fraction gradient all models show two differentiated environments separated by a transition region: the "PDR" $\left(A_{\mathrm{V}} \simeq 0-2\right)$ and the "shielded core" $\left(A_{\mathrm{V}} \gtrsim 6\right)$. The electron density at every cloud position is given by the difference of cations and anions densities;

$n_{\mathrm{e}}=\sum_{i} n_{i}\left(\right.$ cations $\left.^{+}\right)-\sum_{j} n_{j}\left(\right.$ anions $\left.^{-}\right)$.

In the PDR, carbon, the most abundant heavy element with a ionization potential below $13.6 \mathrm{eV}$, provides most of the charge: $n\left(\mathrm{e}^{-}\right) \simeq n\left(\mathrm{C}^{+}\right)$. Therefore, the ionization fraction in the PDR is high, $\left[\mathrm{e}^{-}\right] \sim 10^{-4}$, and independent of elemental abundances other than carbon.

As $\mathrm{A}_{\mathrm{V}}$ increases inwards the cloud, the $\mathrm{C}^{+}$abundance decreases by several orders of magnitude and so does the abundance of electrons. In the shielded core $\left(A_{\mathrm{V}} \gtrsim 6\right)$, low ionization heavy metal ions (e.g., $\mathrm{Fe}^{+}, \mathrm{Mg}^{+}$or $\mathrm{Na}^{+}$) determine much of the ionization fraction (Oppenheimer \& Dalgarno 1974; Guélin et al. 1982). In the absence of PAHs, abundant molecular ions $m^{+}$transfer charge rapidly to heavy metal atoms $M$ through $m^{+}+M \rightarrow m+M^{+}$reactions. Metal ions recombine orders of magnitude slower than molecular ions (Table 4), and thus a large fraction of them is kept ionized (higher [M] implies higher electron abundances). Therefore, the ionization fraction in the core is highly dependent on the adopted metallicity, and varies from a few $\times 10^{-9}$ for $[\mathrm{M}]=10^{-9}$, to a few $\times 10^{-7}$ for $[\mathrm{M}]=10^{-6}$.

\subsection{The role of $\mathrm{PAHs}$}

Depending on their abundances, the presence of PAHs can alter the chemistry and the ionization balance in dense clouds (e.g., Lepp \& Dalgarno 1988). For our adopted abundance of [PAH $]=10^{-7}$ the right and left panels in Fig. 4 shows that the presence of PAHs most modifies the ionization fraction at $A_{V} \gtrsim 2$. Hence, if not all PAHs accrete onto bigger grains or coagulate towards cloud interiors, $\mathrm{PAH}^{-}$can be abundant through the cloud because the radiative electron attachment rate

$\mathrm{PAH}+\mathrm{e}^{-} \rightarrow \mathrm{PAH}^{-}+h v$

is high $\left(\geq 10^{-7} \mathrm{~cm}^{3} \mathrm{~s}^{-1}\right)$, although probably dependent on the PAH size (Omont 1986; Allamandola et al. 1989; Flower et al. 2007; Wakelam \& Herbst 2008). In the shielded core $\mathrm{PAH}^{-}$is destroyed by recombination with atomic $\left(\mathrm{M}^{+}, \ldots\right)$ and molecular cations $\left(\mathrm{HCO}^{+}, \mathrm{H}_{3} \mathrm{O}^{+}, \ldots\right)$ which are orders of magnitude less abundant than the available cations in the PDR $\left(\mathrm{C}^{+}, \mathrm{S}^{+}, \ldots\right)$. Negative PAH ions thus reach high abundances $\left(\left[\mathrm{PAH}^{-}\right] \simeq 2 \times 10^{-8}\right)$. For our choice of PAH parameters, this means that $\mathrm{PAH}^{-}$can be the most abundant negatively charged species, more than electrons for $A_{\mathrm{V}} \geq 5$. In addition, recombination of atomic ions on $\mathrm{PAH}^{-}$is by far more efficient than the slow radiative recombination on electrons. This is a very important point since heavy metal ions and molecular ions are now neutralized at similar rates. As a result, both the abundance of metal cations and the ionization fraction decreases when PAHs are included, while molecular ions such as $\mathrm{H}^{13} \mathrm{CO}^{+}$ and $\mathrm{DCO}^{+}$increase their abundances (see Fig. 4 right panels). 


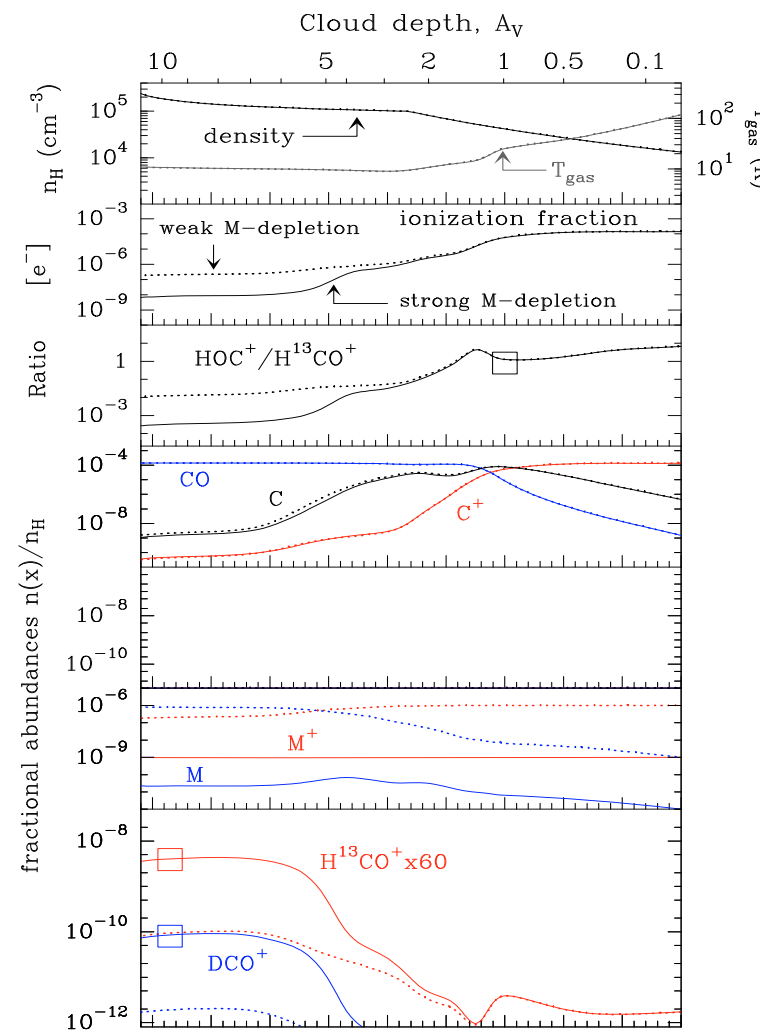

No PAHs; low CR rate No PAHs; high CR rate

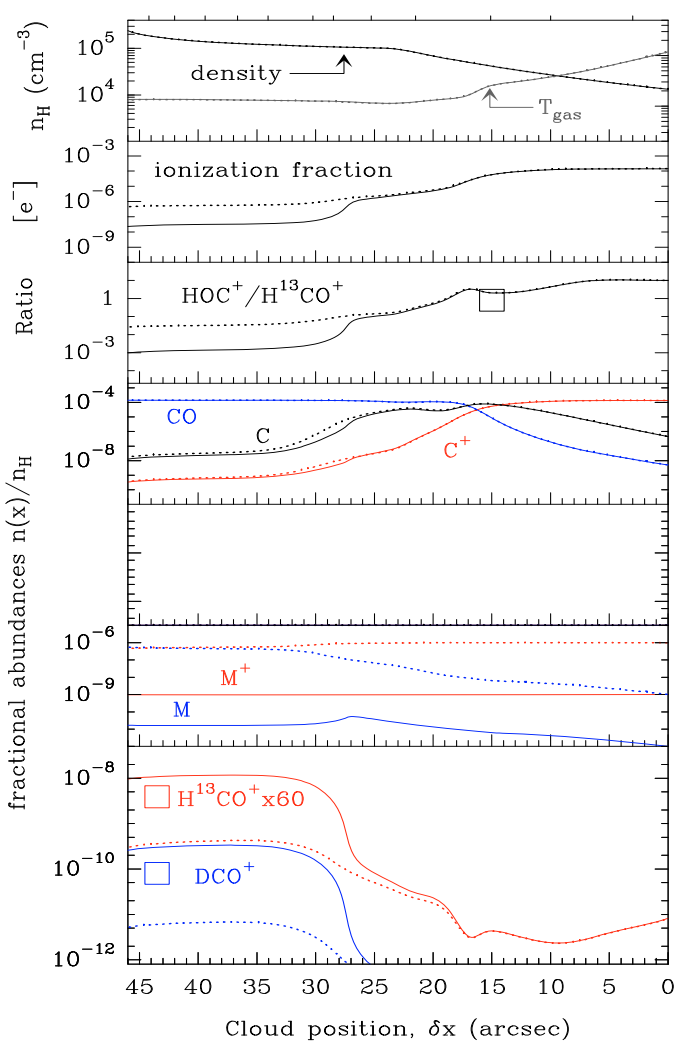

Cloud depth, $A_{\mathrm{V}}$

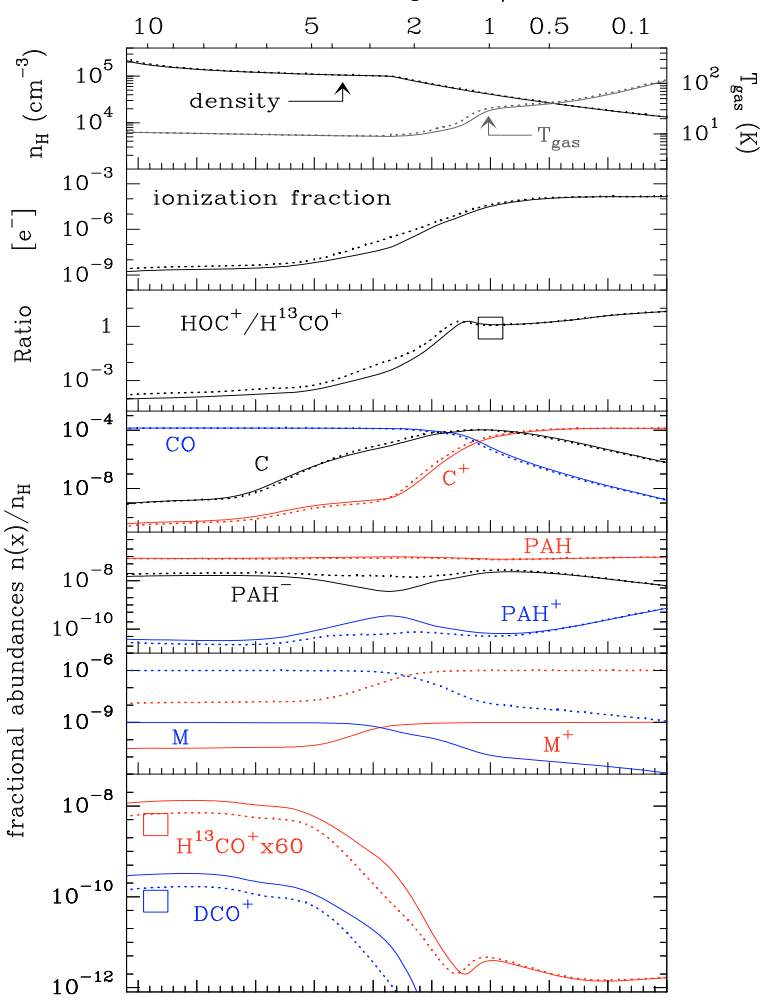

With PAHs; low CR rate With PAHs; high CR rate

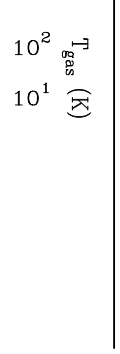

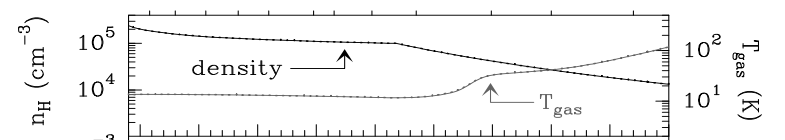

\section{远}



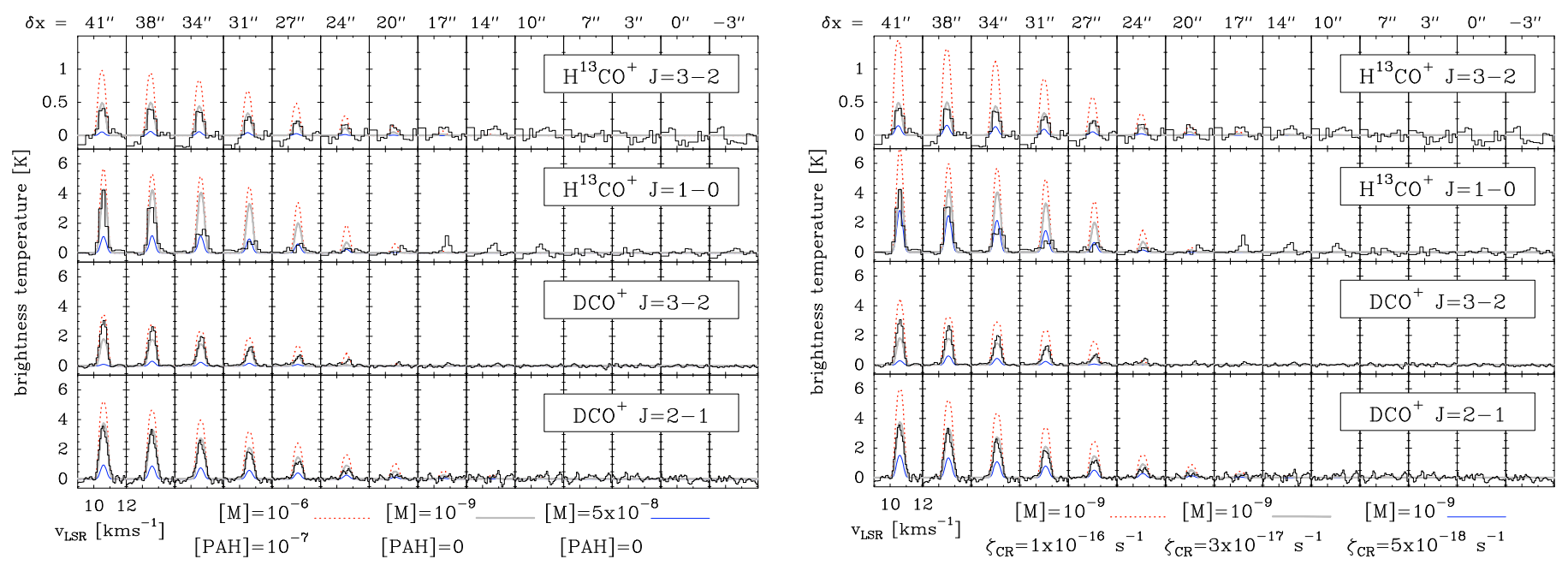

Fig. 5. Left: $\mathrm{H}^{13} \mathrm{CO}^{+}$and $\mathrm{DCO}^{+}$spectra along the direction of the exciting star at $\delta y=15^{\prime \prime}$ (histograms). Radiative transfer models using the output of PDR models for a fixed cosmic-ray ionization rate $\left(\zeta=3 \times 10^{-17} \mathrm{~s}^{-1}\right)$ and varying metallicities. Thin blue curves for $[\mathrm{M}]=5 \times 10^{-8}$ and no PAHs; thick grey curves for $[\mathrm{M}]=10^{-9}$ and no PAHs; dashed red curves for $[\mathrm{M}]=10^{-6}$ and $[\mathrm{PAH}]=10^{-7}$. Modeled line profiles have been convolved with the appropriate Gaussian beam at each observed frequency (the angular resolution for each line are quoted in Tables 1 and 2). Right: same as left but for a fixed metal abundance $\left([\mathrm{M}]=10^{-9}\right)$, no PAHs and varying cosmic-ray ionization rate $\zeta$. Thin blue curves for a model with $\zeta=5 \times 10^{-18} \mathrm{~s}^{-1}$; thick grey curves for $\zeta=3 \times 10^{-17} \mathrm{~s}^{-1}$; dashed red curves for $\zeta=10^{-16} \mathrm{~s}^{-1}$.

At the illuminated edge of the cloud, $\mathrm{PAH}^{-}$is predominantly destroyed by UV photons through electron detachment,

$\mathrm{PAH}^{-}+h v \rightarrow \mathrm{PAH}+\mathrm{e}^{-}$

and through recombination with atomic cations which are very abundant in the PDR (e.g., Bakes \& Tielens 1998; Wolfire et al. 2008). As a consequence, the abundance of ions such as $\mathrm{S}^{+}$in the PDR decreases with respect to models without PAHs. This effect is important to determine the elemental abundances and depletion factors. Despite the higher $\mathrm{PAH}^{-}$destruction rates in the PDR, the high electron density and relatively low UV field in the Horsehead allows $\mathrm{PAH}^{-}$to form efficiently through electron attachment (reaction 11). Hence the resulting $\mathrm{PAH}^{-}$abundance is also high in the PDR. On the other hand, the predicted abundance of positively charged $\mathrm{PAH}^{+}$in our models is $\sim 500$ times smaller than the abundance of $\mathrm{PAH}^{-}$(due to fast electronic recombination) and therefore $\mathrm{PAH}^{+}$do not seem to play a major role in the ionization balance inside the cloud (see also Lepp \& Dalgarno 1988; Wakelam \& Herbst 2008).

\subsection{The role of the cosmic-ray ionization rate}

Cosmic rays affect the ionization state and the physics of molecular clouds, being the dominant source of heating and ionization in the gas shielded from interstellar radiation fields. Indeed, secondary UV photons are created in cloud interiors by $\mathrm{H}_{2}$ electron cascades following $\mathrm{H}_{2}$ excitation by collisions with cosmic rays (Prasad \& Tarafdar 1983). Therefore, cosmic rays maintain a certain ionization degree in the shielded gas and play a fundamental role in the ion-neutral chemistry by setting the abundance of key ions (Herbst \& Klemperer 1973).

Most studies based on the interpretation of observed molecular ions set a range of a few $10^{-17}$ to a few $10^{-16} \mathrm{~s}^{-1}$ for the cosmic-ray ionization rate (Le Petit et al. 2004; van der Tak 2006; Dalgarno 2006 and references therein). However, it is still debated whether or not $\zeta$ depends on environmental conditions (e.g., galactic center vs. disk clouds) or if it varies from source to source (e.g., from dense molecular cores to more translucent clouds). In many ways, PDRs offer an interesting intermediate medium to analyze the transition between translucent and dark clouds.

In terms of our observations, the $\mathrm{DCO}^{+}$and $\mathrm{H}^{13} \mathrm{CO}^{+}$abundances directly scale with $\zeta$ in the UV shielded gas. Indeed, these ions are direct products of the $\mathrm{H}_{3}^{+}$destruction (through reactions 2 and 3), and the $\mathrm{H}_{3}^{+}$formation is proportional to $\simeq \zeta n_{\mathrm{H}}$. However, $\zeta$ and the metal abundance cannot be constrained independently from the inferred $\mathrm{DCO}^{+}$and $\mathrm{H}^{13} \mathrm{CO}^{+}$abundances since both parameters control the ionization fraction, and thus the destruction of these ions through reactions 5 and 6 .

\section{Results: observational constraints}

In this section we compare the synthetic and observed $\mathrm{H}^{13} \mathrm{CO}^{+}$ and $\mathrm{DCO}^{+}$spectra as a function of cloud position. We then explore the range of metallicities and cosmic-ray ionization rates compatible with the $\mathrm{H}^{13} \mathrm{CO}^{+}$and $\mathrm{DCO}^{+}$inferred abundances (see Table 6). The influence of PAHs is also investigated. We finally compare the $\left[\mathrm{HOC}^{+}\right] /\left[\mathrm{H}^{13} \mathrm{CO}^{+}\right]$ratio obtained towards the Horsehead with the values derived in other PDRs.

\subsection{Constraints to the metals abundance}

Figure 5 left shows the spectra along the direction of the exciting star (histograms) and radiative transfer models using the output of several PDR models for a fixed ionization rate $(\zeta=3 \times$ $\left.10^{-17} \mathrm{~s}^{-1}\right)$ and varying metallicities. In particular, the model with $[\mathrm{M}]=10^{-9}$ (and no PAHs) displays a notable agreement with both the $\mathrm{DCO}^{+}$and $\mathrm{H}^{13} \mathrm{CO}^{+}$spatial distribution and with the inferred peak abundances towards the core (Table 6). In addition, Fig. 6 left shows the predicted ionization fraction and $\left[\mathrm{H}^{13} \mathrm{CO}^{+}\right]$ and $\left[\mathrm{DCO}^{+}\right]$abundances at the core peak $\left(A_{V}>10\right)$ as a function of [M] (blue-solid curves). These models (no PAHs, fixed $\zeta$ ) show that the upper limit metallicity compatible with observations is $[\mathrm{M}] \leq 4 \times 10^{-9}$, which implies an ionization fraction of $\left[\mathrm{e}^{-}\right]=(7 \pm 1) \times 10^{-9}$ at the core peak. Higher metal abundances increase the ionization fraction (see Fig. 6 left), which translates into weaker lines than observed (Fig. 5 left: thin-blue curves). Therefore, the gas-phase metal abundance is depleted by $\sim 4$ 

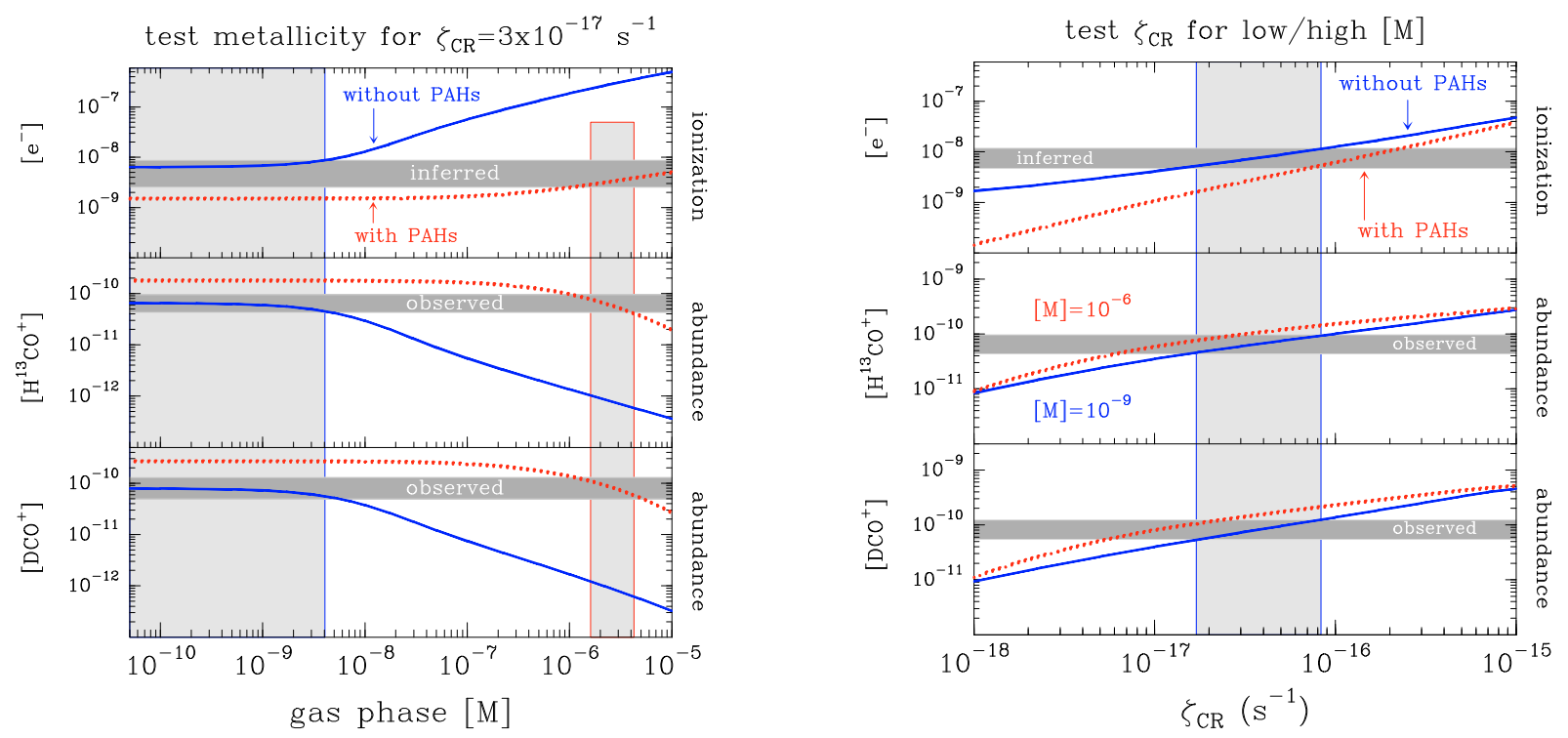

Fig. 6. Left: model predictions for the shielded core (the " $\mathrm{DCO}^{+}$peak" at $A_{\mathrm{V}}>10$ ). The ionization rate due to cosmic rays is fixed to $\zeta=$ $3 \times 10^{-17} \mathrm{~s}^{-1}$. The different panels show (upper): the ionization fraction, (middle): the $\mathrm{H}^{13} \mathrm{CO}^{+}$abundance and (lower): the $\mathrm{DCO}^{+}$abundance as a function of gas phase metallicity. Blue-solid curves for models without PAHs, and red-dotted curves for models with neutral and charged PAHs $\left([\mathrm{PAH}]=10^{-7}\right)$. Horizontal shaded regions show the $\mathrm{H}^{13} \mathrm{CO}^{+}$and $\mathrm{DCO}^{+}$abundances derived from observations towards the core, while vertical shaded regions show the parameter space compatible with observations. Right: same as previous figure but for a fixed low metallicity of $[\mathrm{M}]=10^{-9}$ (no PAH; blue-solid curves) and a fixed high metallicity of $[\mathrm{M}]=10^{-6}$ ([PAH] $=10^{-7}$; red-dotted curves). The different panels show (upper): the ionization fraction, (middle): the $\mathrm{H}^{13} \mathrm{CO}^{+}$abundance and (lower): the $\mathrm{DCO}^{+}$abundance as a function of the ionization rate due to cosmic rays.

Table 6. Inferred abundances $[\mathrm{x}]=N(\mathrm{x}) / N_{\mathrm{H}}$ where $N_{\mathrm{H}}=N(\mathrm{H})+2 N\left(\mathrm{H}_{2}\right)$.

\begin{tabular}{lcc}
\hline \hline Species & $\begin{array}{c}\text { Shielded core } \\
A_{\mathrm{V}} \geq 6\end{array}$ & PDR \\
& $\delta x \simeq 45^{\prime \prime}$ & $\begin{array}{c}A_{\mathrm{V}}=0-2 \\
\delta x \simeq 15^{\prime \prime}\end{array}$ \\
\hline$N_{\mathrm{H}}\left(\mathrm{cm}^{-2}\right)$ & $5.8 \times 10^{22}$ & $3.1 \times 10^{22}$ \\
{$\left[\mathrm{H}^{13} \mathrm{CO}^{+}\right]$} & $6.5 \times 10^{-11}$ & $1.5 \times 10^{-11}$ \\
{$\left[\mathrm{H}^{12} \mathrm{CO}^{+}\right]$} & $3.9 \times 10^{-9}$ & $9.0 \times 10^{-10}$ \\
{$\left[\mathrm{DCO}^{+}\right]$} & $8.0 \times 10^{-11}$ & $(-)$ \\
{$\left[\mathrm{HOC}^{+}\right]$} & $(-)$ & $0.4 \times 10^{-11} \dagger$ \\
{$\left[\mathrm{CO}^{+}\right]$} & $(-)$ & $\leq 5.0 \times 10^{-13}$ \\
{$\left[\mathrm{e}^{-}\right]$} & $(1-8) \times 10^{-9}$ & $10^{-6}-10^{-4}$ \\
\hline
\end{tabular}

$\dagger$ Assuming extended emission. It would be $1.2 \times 10^{-11}$ if $\mathrm{HOC}^{+}$arises from a $12^{\prime \prime}$-width filament as HCO (Gerin et al. 2009).

orders of magnitude with respect to the Sun $\left([\mathrm{M}] \simeq 8.5 \times 10^{-5}\right.$, Anders \& Grevesse 1989). This range of depletion is similar to that obtained in other prestellar cores such as Barnard 68 (Maret \& Bergin 2007). We shall refer it as the strong metal depletion case.

The inclusion of PAH interactions implies lower ionization fractions and enhanced molecular ion abundances (see Fig. 6 left) which result in overestimated $\mathrm{H}^{13} \mathrm{CO}^{+}$and $\mathrm{DCO}^{+}$line intensities towards the core. Therefore, the abundance of metals (e.g., indirectly the ionization fraction) has to be increased to match the observed intensities. In particular, Fig. 5 left shows that a model with $[\mathrm{PAHs}]=10^{-7}$ and $[\mathrm{M}]=10^{-6}$ (red-dashed curves) displays only a factor $<2$ brighter lines than models with 3 orders of magnitude lower metallicities and no PAHs (thickgrey curves). As shown in Fig. 6 left, the inclusion of PAHs makes the range of metal abundances compatible with observations much higher now, $[\mathrm{M}]=(3 \pm 1) \times 10^{-6}$. The required abundance of metals is at least a factor $\sim 500$ larger than the former case without PAHs. Thus, we refer to it as the weak metal depletion case. Note that this metallicity is still below the gasphase abundance of $\mathrm{Fe}+\mathrm{Mg}+\ldots$ elements in the diffuse interstellar gas (e.g., Wolfire et al. 1995; Howk et al. 2006) and is consistent with the incorporation of metals into dust grains in higher density regions (e.g., Wolfire et al. 1995). On the other hand, the ionization fraction required to reproduce the $\mathrm{H}^{13} \mathrm{CO}^{+}$and $\mathrm{DCO}^{+}$ abundances does not change much, $\left[\mathrm{e}^{-}\right]=(4 \pm 1) \times 10^{-9}$ at the core peak (red-dashed curves Fig. 6 left). Therefore, it is not easy to distinguish between the strong metal depletion (no PAHs) and weak metal depletion (with PAHs) cases in terms of the ionization fraction. The observation of forbidden lines from metals such as [Fe II] towards the UV illuminated edges of molecular clouds may help to remove this apparent degeneracy. In one of the few positive cases, the S140 PDR, the detection of a weak $[\mathrm{Fe}$ II $] 26.0 \mu \mathrm{m}$ fine-structure line emission suggests that iron is depleted, but with an abundance of $\sim 5 \times 10^{-8}$ relative to H (Timmermann et al. 1996). Nevertheless, without mapping and comparing with other PDR tracers, it is not obvious to disentangle whether [Fe II] lines arise from the PDR gas or from the adjacent (HII) ionization front (e.g., Marconi et al. 1998; Kaufman et al. 2006).

\subsection{Constraints to the cosmic-ray ionization rate}

Figure 5 right shows again the observed spectra along the direction of the exciting star (histograms) and radiative transfer models using the output of PDR models that vary the cosmic ray rate, without PAHs and a fixed metal abundance of $[\mathrm{M}]=10^{-9}$. The adopted metal abundance is, within an order of magnitude, the usual value estimated in prestellar cores (e.g., Caselli et al. 1999; Maret \& Bergin 2007) and is compatible with our strong metal depletion case. Therefore, Fig. 5 right shows the effects of different ionization rates directly on the $\mathrm{DCO}^{+}$and $\mathrm{H}^{13} \mathrm{CO}^{+}$line intensities. For the adopted physical conditions and chemical network, our observations constrain $\zeta$ within a factor $\sim 2$. In 

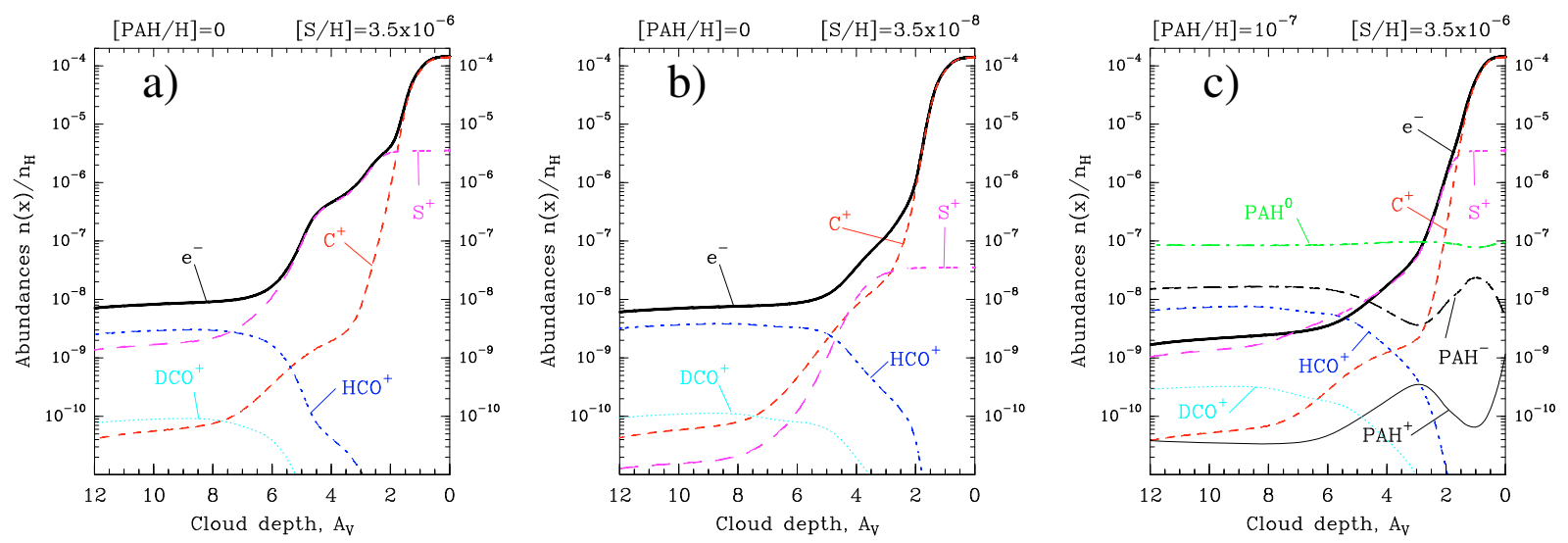

Fig. 7. Derived abundance profiles for the most significant ions studied in this work for different PAH and sulfur elemental abundances. An enhanced UV radiation field 60 times the mean ISRF illuminates the cloud from the right. The metal abundance $\left([\mathrm{M}]=10^{-9}\right)$ and the cosmic-ray rate $\left(\zeta=3 \times 10^{-17} \mathrm{~s}^{-1}\right)$ are fixed in all models. a) Model with $[\mathrm{PAH}]=0$ and $[\mathrm{S}]=3.5 \times 10^{-6}$ (low sulfur depletion). b) Model with $[\mathrm{PAH}]=0$ and $[\mathrm{S}]=3.5 \times 10^{-8}$ (high sulfur depletion). c) Model with $[\mathrm{PAH}]=10^{-7}$ and $[\mathrm{S}]=3.5 \times 10^{-6}$.

particular, Fig. 6 right shows the predicted $\left[\mathrm{e}^{-}\right],\left[\mathrm{H}^{13} \mathrm{CO}^{+}\right]$and $\left[\mathrm{DCO}^{+}\right]$abundances at the core peak $\left(A_{V}>10\right)$ as a function of $\zeta$, and suggests that in the absence of PAHs, the cosmic ray ionization rate range compatible with the observations of the Horsehead edge is $\zeta=(5 \pm 3) \times 10^{-17} \mathrm{~s}^{-1}$ (solid-blue curves). If PAHs are included in the chemistry, the metal abundance has to be increased accordingly to reproduce the observations. For our $[\mathrm{PAH}]=10^{-7}$ model case, the required metal abundance needs to be above $[\mathrm{M}] \simeq 10^{-6}$ to obtain ionization rates above $\zeta \simeq 10^{-17} \mathrm{~s}^{-1}$ (Fig. 6 right; red-dotted curves).

Note that given the fact that the $\mathrm{H}^{13} \mathrm{CO}^{+}$formation in the PDR is not dominated by the ${ }^{13} \mathrm{CO}+\mathrm{H}_{3}^{+}$reaction, the $\mathrm{H}^{13} \mathrm{CO}^{+}$ abundance in the UV illuminated gas does not scale with $\zeta$. Therefore, we cannot further investigate if $\zeta$ varies significantly in the transition from diffuse regions to the shielded core (e.g., McCall et al. 2003; Padoan \& Scalo 2005).

\subsection{High ionization fraction in the PDR}

The bright $[\mathrm{C}$ II $] 158 \mu \mathrm{m}$ (Zhou et al. 1993) and [C I] $492 \mathrm{GHz}$ (Philipp et al. 2006) fine structure line emission towards the Horsehead PDR, together with subtle chemical effects such as the large $\left[l-\mathrm{C}_{3} \mathrm{H}_{2}\right] /\left[c-\mathrm{C}_{3} \mathrm{H}_{2}\right]$ linear-to-cyclic abundance ratio (Teyssier et al. 2005) all show observationally that the ionization fraction is higher in the UV illuminated edge than towards the cloud interior. Nevertheless, all those studies lacked the angular resolution to properly measure the ionization fraction gradient.

The abundances of reactive ions such as $\mathrm{HOC}^{+}$are also predicted to be enhanced in the UV illuminated gas, where we have shown that the ionization fraction is high, up to $\left[\mathrm{e}^{-}\right] \sim 10^{-4}$, and that the $\mathrm{HOC}^{+}$formation is linked to the availability of $\mathrm{C}^{+}$. On the other hand, the $\mathrm{H}^{13} \mathrm{CO}^{+}$abundance increases as the electron abundance decreases towards the shielded core. Therefore, we predict that the $\left[\mathrm{HOC}^{+}\right] /\left[\mathrm{H}^{13} \mathrm{CO}^{+}\right]$ abundance ratio scales with the ionization fraction gradient, reaching the highest values in the PDR (Fig. 4). In particular, we derive a high $\left[\mathrm{HOC}^{+}\right] /\left[\mathrm{H}^{13} \mathrm{CO}^{+}\right]=0.3-0.8$ ratio (or a low $\left[\mathrm{HCO}^{+}\right] /\left[\mathrm{HOC}^{+}\right] \simeq 75-200$ ratio) towards the PDR, similar to that observed in other PDRs such as NGC 7023 (Fuente et al. 2003).

\section{Discussion}

\subsection{The ionization fraction gradient}

Star forming clouds display different environments depending on the dominant physical and chemical processes. These environments are, to a first approximation, similar to those studied here: (i) a low density cloud edge directly exposed to a UV radiation field from nearby stars; (ii) a transition region or ridge where the $\mathrm{H}_{2}$ density increases as the gas temperature decreases due to the attenuation of the external radiation field. UV photons can still play a significant role depending on their penetration depths (e.g., cloud clumpiness, grain properties, etc.); and (iii) the denser shielded cores that may be externally triggered to form a new generation of stars depending on their stability against gravitational collapse (e.g., Goicoechea et al. 2008).

Assuming that the observed field-of-view in the Horsehead nebula is representative of the above 3 environments, our maps and chemical models reveal that the ionization fraction follows a steep gradient in molecular clouds: from $\left[\mathrm{e}^{-}\right] \simeq 10^{-4}$ at the edge of the cloud (the " $\mathrm{C}^{+}$dominated" region) to a few times $\sim 10^{-9}$ in the shielded cores. The prevailing chemistry and the abundance of atomic ions such as $\mathrm{C}^{+}$and $\mathrm{S}^{+}$determine the "slope" of the ionization fraction gradient in the transition regions. In particular, sulfur (with a ionization potential of $\sim 10.36 \mathrm{eV}$ ) is a good source of charge behind the " $\mathrm{C}^{+}$dominated" region. Advection and time-dependent effects may also modify the ionization fraction gradient with time. However, Morata \& Herbst (2008) have shown models for (uniform) physical conditions similar to those in the Horsehead where $\left[\mathrm{HCO}^{+}\right]$(and $\left[\mathrm{e}^{-}\right]$presumably) does not change with time appreciably.

Figure 7 shows the predicted ionization fraction gradient, with a scale length of $\sim 0.05 \mathrm{pc}$ ( or $\sim 25^{\prime \prime}$ ), and the main charge carriers for 3 representative models with fixed standard metal abundance (our strong metal depletion case) and standard cosmic-ray rate. Panel $7 a$ shows a model without PAHs and high gas-phase sulfur abundance $([\mathrm{S}]=3.5 \times$ $10^{-6}$; Goicoechea et al. 2006). The ionization fraction gradient in the core/transition/edge zones, is mainly determined by the $\left[\mathrm{HCO}^{+}+\mathrm{S}^{+}+\mathrm{M}^{+}+\ldots\right] /\left[\mathrm{S}^{+}\right] /\left[\mathrm{C}^{+}\right]$abundances respectively. Sulfur ions control the charge balance in the transition layers, and due to their high abundance and slow radiative recombination rate with electrons, the ionization fraction is high, a few times $10^{-7}$, and the gradient is smooth. This model qualitatively agrees with the observed more extended emission and narrower 
line-widths of sulfur recombination lines compared to carbon recombination lines in dark clouds, as well as with the relatively low $(<10)$ carbon-to-sulfur recombination lines intensity ratio (e.g., Pankonin \& Walmsley 1978; Falgarone et al. 1978). All these signatures argue in favor of extended regions with significant amounts of gas-phase $\mathrm{S}^{+}$.

Panel $7 b$ shows the same model but sulfur abundances smaller by two orders of magnitude (strong gas-phase sulfur depletion). The ionization fraction in the cloud core $\left(A_{\mathrm{V}}>6\right)$ and edge $\left(A_{\mathrm{V}}<2\right)$ are nearly the same as in the previous high sulfur abundance model. However, the lack of abundant $\mathrm{S}^{+}$in the transition layers decreases the electron abundance considerably, and makes the ionization fraction gradient much steeper. Observational constraints to the atomic and ionic $\mathrm{S}$ abundances from far-IR fine structure lines or recombination lines, and a careful treatment of the sulfur chemistry (i.e., which are the most abundant S-bearing species as a function of cloud depth?) are thus required to quantify the $\mathrm{S}^{+}$abundance at large $\mathrm{A}_{V}$ and its impact on the charge balance.

Panel $7 c$ shows a model with high sulfur abundance but including PAHs (with $[\mathrm{PAH}]=10^{-7}$ ). As presented in Sect. 5.2, negatively charged $\mathrm{PAH}^{-}$efficiently form by radiative electron attachment and their abundance remains high through the cloud. Given the much higher recombination rates of atomic ions on $\mathrm{PAH}^{-}$than on electrons, the abundance of atomic ions such as $\mathrm{S}^{+}$in the transition zone, or $\mathrm{M}^{+}$in the shielded cores, quickly decreases. Hence, lower ionization fractions (and a much weaker dependence on the assumed metal elemental abundance) are predicted by the model with PAHs. These results agrees with theoretical predictions for UV shielded gas (Lepp \& Dalgarno 1988; Flower et al. 2007; Wakelam \& Herbst 2008).

In summary, a high abundance of PAHs throughout the molecular cloud (not only in the PDR) plays a role in the ionization balance and in the abundance of molecular ions, which affects the determination of elemental abundances (e.g., S) from fractional molecular abundances (e.g., $\mathrm{HCS}^{+} / \mathrm{CS}, \mathrm{SO}^{+} / \mathrm{SO}$, etc.).

\subsection{The PAH abundance in UV shielded gas}

The PAH abundance in the dense and UV shielded gas is far from being well constrained. Different approaches to analyze ISO and Spitzer mid-IR observations towards several PDRs all argue in favor of an evolution of dust grain sizes: from the illuminated cloud edge where the emission of PAH bands dominates, to the shielded interiors where the continuum emission from bigger grains dominates (Rapacioli et al. 2005; Berné et al. 2007; Compiègne et al. 2008). It is not trivial to disentangle whether this is a physical effect (i.e., free PAHs are not present in the shielded regions) or an excitation effect (i.e., lack of UV photons). Even if the PAH abundance drastically decreases towards cloud interiors, a chemically significant fraction of them may survive. Unfortunately, while the effects of grain growth in the UV extinction curve have been modelled by us (Goicoechea \& Le Bourlot 2007), including PAH coagulation/accretion in the chemistry is beyond the scope of this work. All we can say at this point is that a better description of the cloud chemistry may be a decreasing PAH abundance gradient or an increasing PAH size distribution towards the cloud interior. In any case, we have shown that the presence of free PAHs in molecular clouds modifies the prevailing chemistry. As a result, the predicted high abundance of $\mathrm{PAH}^{-}$can dominate the recombination of metal ions and reduce the ionization fraction.

The presence of abundant free PAHs, or large molecules to which electron attach (Lepp \& Dalgarno 1988), can thus be crucial in determining the coupling of the gas with magnetic fields in molecular clouds, but also in collapsing cores or in the "dead" zones of protoplanetary disks (magnetically inactive regions where accretion cannot occur if the ionization fraction is very low). According to our models, the PAH abundance threshold required to affect the metal and electron abundance determination in the UV shielded gas is $[\mathrm{PAH}]>10^{-8}$. Herschel observations might allow the identification of specific PAH carriers through their far-IR skeletal modes (Joblin et al. 2002; Mulas et al. 2006), thus providing clues to their composition and abundance variations in different environments.

\section{3. "Non standard" $\mathrm{HCO}^{+}$dissociative recombination rate}

We conclude by discussing the sensitivity of our determination of the ionization fraction from $\mathrm{H}^{13} \mathrm{CO}^{+}$and $\mathrm{DCO}^{+}$abundances. In particular, we have checked the effects of adopting a smaller, "non standard" $\mathrm{HCO}^{+}$dissociative recombination rate, $\alpha^{\prime}\left(\mathrm{HCO}^{+}\right)=0.7 \times 10^{-7}(300 / T)^{0.50} \mathrm{~cm}^{3} \mathrm{~s}^{-1}$ (Sheehan 2000, Florescu-Mitchell \& Mitchell 2006). For models without PAHs, the predicted $\mathrm{H}^{13} \mathrm{CO}^{+}$and $\mathrm{DCO}^{+}$abundances increase by a factor of $\sim 3$ with respect to models using the "standard" $\alpha\left(\mathrm{HCO}^{+}\right)$ rate (Table 4), but the metallicity required to fit the observed lines has to be increased to $[\mathrm{M}] \simeq 5 \times 10^{-8}$ and the predicted ionization fraction increases to $\left[\mathrm{e}^{-}\right] \simeq 5 \times 10^{-8}$ in the core. This value should be regarded as the upper limit of our determination. On the other hand, the influence of $\alpha^{\prime}\left(\mathrm{HCO}^{+}\right)$in models with PAHs is less important. It also requires high metallicities to fit the observed intensities (weak metal depletion case), but the predicted $\left[\mathrm{e}^{-}\right]$in the shielded core remains low (below $\sim 10^{-8}$ ).

\section{Summary and conclusions}

We have presented the first detection of $\mathrm{HOC}^{+}$reactive ion towards the Horsehead PDR. Combined with our previous IRAMPdBI $\mathrm{H}^{13} \mathrm{CO}^{+} J=1-0$ (Gerin et al. 2009) and IRAM-30 $\mathrm{m}$ $\mathrm{H}^{13} \mathrm{CO}^{+}$and $\mathrm{DCO}^{+}$higher- $J$ lines maps (Pety et al. 2007) we performed a detailed analysis of their chemistry, excitation and radiative transfer to constrain the ionization fraction as a function of cloud position. The observed field contains 3 different environments: (i) the UV illuminated cloud edge; (ii) a transition region or ridge; and (iii) a dense and cold shielded core. We have presented a study of the ionization fraction gradient in the above environments, which can be considered as templates for most molecular clouds. Our main conclusions are the following:

1. The ionization fraction follows a steep gradient, with a scale length of $\sim 0.05 \mathrm{pc}\left(\sim 25^{\prime \prime}\right)$, from $\left[\mathrm{e}^{-}\right] \simeq 10^{-4}\left(n_{\mathrm{e}} \sim 1-5 \mathrm{~cm}^{-3}\right)$ at the cloud edge (the " $\mathrm{C}^{+}$dominated" regions) to a few times $\sim 10^{-9}$ in the shielded core (with ongoing deuterium fractionation). Sulfur, metal and PAH ions play a key role in the charge balance at different cloud depths.

2. The detection of $\mathrm{HOC}^{+}$towards the PDR, and the high $\left[\mathrm{HOC}^{+}\right] /\left[\mathrm{H}^{13} \mathrm{CO}^{+}\right] \simeq 0.3-0.8$ abundance ratio inferred, proves the high ionization fraction in the UV irradiated gas. However, the $\mathrm{H}^{13} \mathrm{CO}^{+}$and $\mathrm{HOC}^{+}$abundances derived from observations are larger than the PDR model predictions. We propose that either the gas is/was warmer than predicted or that significant water ice-mantle photodesorption is taking place and $\mathrm{HOC}^{+}$is mainly formed by the $\mathrm{C}^{+}+\mathrm{H}_{2} \mathrm{O}$ reaction.

3. The ionization fraction in the shielded core depends on the metal abundance and on the cosmic-ray ionization rate. Assuming a standard rate of $\zeta=3 \times 10^{-17} \mathrm{~s}^{-1}$ and pure gasphase chemistry (no PAHs), the metal abundance has to be 
lower than $4 \times 10^{-9}$ (strong metal depletion). Conversely, assuming a standard metal abundance of $[\mathrm{M}]=10^{-9}$, our observations can only be reproduced with $\zeta=(5 \pm 3) \times 10^{-17} \mathrm{~s}^{-1}$.

4. The inclusion of PAHs modifies the ionization fraction gradient and decreases the metal depletion required to reproduce the observations if $[\mathrm{PAH}]>10^{-8}$ (i.e., if not all PAHs coagulate/accrete onto bigger grains). In such a case, $\mathrm{PAH}^{-}$acquire large abundances also in the shielded gas. Recombination of atomic ions on $\mathrm{PAH}^{-}$is much more efficient than on electrons and thus metal ions and molecular ions are neutralized at similar rates. For $[\mathrm{PAH}]=10^{-7}$, the metal abundance consistent with observations increases to $[\mathrm{M}]=(3 \pm 1) \times 10^{-6}$ (still below the heavy metals abundance in the diffuse ISM).

Acknowledgements. We thank the IRAM staff for their support during observations and D. Talbi and B. Godard for useful advice regarding the $\mathrm{HCO}^{+}$dissociative recombination rate. Inelastic collisional rates of $\mathrm{HCO}^{+}$with electrons were kindly provided by A. Faure and J. Tennyson. We also thank M. Walmsely for several interesting comments. We acknowledge the use of OSU (http://www . physics.ohio-state.edu/ eric/research.html) and UDFA (http:// www.udfa.net/) chemical reaction databases. We finally acknowledge financial support from CNRS/INSU research programme PCMI. JRG is supported by a Ramón y Cajal research contract from the Spanish MICINN and co-financed by the European Social Fund.

\section{References}

Abergel, A., Teyssier, D., Bernard, J. P., et al. 2003, A\&A, 410, 577 Allamandola, L. J., Tielens, A. G. G. M., \& Barker, J. R. 1985, ApJ, 290, L25 Allamandola, L. J., Tielens, A. G. G. M., \& Barker, J. R. 1989, ApJS, 71, 733 Anders, E., \& Grevesse, N. 1989, Geochim. Cosmochim. Acta, 53, 197

Bakes, E. L. O., \& Tielens, A. G. G. M. 1994, ApJ, 427, 822

Bakes, E. L. O., \& Tielens, A. G. G. M. 1998, ApJ, 499, 258

Balbus, S. A., \& Hawley, J. F. 1991, ApJ, 376, 214

Berné, O., Joblin, C., Deville, Y., et al. 2007, A\&A, 469, 575

Boulanger, F., Falgarone, E., Puget, J. L., \& Helou, G. 1990, ApJ, 364, 136

Caselli, P., Walmsley, C. M., Terzieva, R., \& Herbst, E. 1998, ApJ, 499, 234

Caselli, P., Walmsley, C. M., Tafalla, M., Dore, L., \& Myers, P. C. 1999, ApJ, 523, L165

Cernicharo, J., \& Guélin, M. 1987, A\&A, 176, 299

Cernicharo, J., Goicoechea, J. R., Pardo, J. R., \& Asensio-Ramos, A. 2006, ApJ, 642,940

Compiègne, M., Abergel, A., Verstraete, L., et al. 2007, A\&A, 471, 205

Compiègne, M., Abergel, A., Verstraete, L., \& Habart, E. 2008, A\&A, 491, 797

Dalgarno, A. 2006, Proceedings of the National Academy of Science, 103, 33, 12269

de Boisanger, C., Helmich, F. P., \& van Dishoeck, E. F. 1996, A\&A, 310, 315

Desert, F.-X., Boulanger, F., \& Puget, J. L. 1990, A\&A, 237, 215.

Falgarone, E., Cesarsky, D. A., Encrenaz, P. J., \& Lucas, R. 1978, A\&A, 65, L13

Faure, A., \& Tennyson, J. 2001, MNRAS, 325, 443

Florescu-Mitchell, A. I., \& Mitchell, J. B. A. 2006, Phys. Rep., 430, 277

Flower, D. R. 1999, MNRAS, 305, 651

Flower, D. R., \& Pineau des Forêts, G. 2003, MNRAS, 343, 390

Flower, D. R., Pineau des Forêts, G., \& Walmsley, C. M. 2007, A\&A, 474, 923

Fuente, A., Rodríguez-Franco, A., García-Burillo, S., Martín-Pintado, J., \& Black, J. H. 2003, A\&A, 406, 899

Fuente, A., García-Burillo, S., Usero, A., et al. 2008, A\&A, 492, 675

Gerin, M., Goicoechea, J. R., Pety, J., \& Hily-Blant, P. 2009, A\&A, in press

Gerlich, D., Herbst, E., \& Roueff, E. 2002, Planetary and Space Science, 50, 12, 1275

Goicoechea, J. R., \& Le Bourlot, J. 2007, A\&A, 467, 1

Goicoechea, J. R., Pety, J., Gerin, M., et al. 2006, A\&A, 456, 565

Goicoechea, J. R., Berné, O., Gerin, M., Joblin, C., \& Teyssier, D. 2008, ApJ, 680,466

Graedel, T. E., Langer, W. D., \& Frerking, M. A. 1982, ApJS, 48, 321

Greve, A., Kramer, C., \& Wild, W. 1998, A\&AS, 133, 271

Guélin, M., Langer, W. D., \& Wilson, R. W. 1982, A\&A, 1982, 107, 107

Habart, E., Abergel, A., Walmsley, C. M., Teyssier, D., \& Pety, J. 2005, A\&A, 437,177

Herbst, E., \& Klemperer, W. 1973, ApJ, 185, 505

Hezareh, T., Houde, M., McCoey, C., Vastel, C., \& Peng, R. 2008, ApJ, 684, 1221
Hily-Blant, P., Teyssier, D., Philipp, S., \& Güsten, R. 2005, A\&A, 440, 909 Hollenbach, D., Kaufman, M. J., Bergin, E. A., \& Melnick, G. J. 2009, ApJ, 690, 1497

Howk, J. C., Sembach, K. R., \& Savage, B. D. 2006, ApJ, 637, 333

Joblin, C., Toublanc, D., Boissel, P., \& Tielens, A. G. G. M. 2002, Mol. Phys., 100,3595

Kaufman, M. J., Wolfire, M. G., \& Hollenbach, D. J. 2006, ApJ, 644, 28

Langer, W. D., \& Penzias, A. A. 1990, ApJ, 357, 477

Langer, W. D., Graedel, T. E., Frerking, M. A., \& Armentrout, P. B. 1984, A\&A, 277,581

Laor, A., \& Draine, B. T. 1993, 402, 441

Le Bourlot, J., Pineau Des Forets, G., Roueff, E., \& Flower, D. R. 1993, A\&A, 267, 233

Leger, A., \& Puget, J. L. 1984, A\&A, 137, L5

Le Petit, F., Roueff, E., \& Herbst, E. 2004, A\&A, 417, 993

Le Petit, F., Nehmé, C., Le Bourlot, J., \& Roueff, E. 2006, ApJS, 64, 506

Lepp, S., \& Dalgarno, A. 1988, ApJ, 324, 553

Li, A., \& Draine, B. T. 2001, ApJ, 554, 778

Liszt, H., Lucas, R., \& Black, J. H. 2004, A\&A, 428, 117L

Marconi, A., Testi, L., Natta, A. \& Walmsley, C. M. 1998, A\&A, 330, 696

Maret, S., \& Bergin, E. A. 2007, ApJ, 664, 956

Mathis, J. S., Rumpl, W., \& Nordsieck, K. H. 1977, ApJ, 217, 425

McCall, B. J., Huneycutt, A. J., Saykally, R. J., et al. 2003, Nature, 422, 500

Morata, O., \& Herbst, E. 2008, MNRAS, 390, 1549

Mulas, G., Malloci, G., Joblin, C., \& Toublanc, D. 2006, A\&A, 460, 93

Neufeld, D. A., Lepp, S., \& Melnick, G. J. 1995, ApJS, 100, 132

Omont, A., 1986, A\&A, 164, 159

Oppenheimer, M., \& Dalgarno, A. 1974, ApJ, 192, 29

Padoan, P., \& Scalo, J. 2005, ApJ, 624, L97

Pankonin, V., \& Walmsley, C. M. 1978, A\&A, 67, 12

Penzias, A. A., \& Burrus, C. A. 1973, ARA\&A, 11, 51

Pety, J., Teyssier, D., Fossé, D., et al. 2005, A\&A, 435, 885

Pety, J. 2005, Semaine de l'Astrophysique Française, meeting held in Strasbourg, France, ed. F. Casoli, T. Contini, J. M. Hameury, \& L. Pagani (EDP Sciences), SF2A-2005, 721

Pety, J., Goicoechea, J. R., Hily-Blant, P., Gerin, M., \& Teyssier, D. 2007, A\&A, 464, L41

Philipp, S. D., Lis, D. C., Güsten, R., Kasemann, C., Klein, T., \& Phillips, T. G. 2006, A\&A, 454, 213

Prasad, S. S., \& Tarafdar, S. P. 1983, ApJ, 267, 603

Rapacioli, M., Joblin, C., \& Boissel, P. 2005, A\&A, 429, 193

Rapacioli, M., Calvo, F., Joblin, C., et al. 2006, A\&A, 460, 519

Rizzo, J. R., Fuente, A., Rodríguez-Franco, A., \& García-Burillo, S. 2003, ApJ, 597, L153

Röllig, M., Abel, N. P., Bell, T., et al. 2007, A\&A, 467, 187

Savage, C., \& Ziurys, L. M. 2004, ApJ, 616, 966

Savage, C., Apponi, A. J., Ziurys, L. M., \& Wyckoff, S. 2000, ApJ, 578, 211

Scott, G. B. I., Fairley, D. A., Freeman, C. G., et al. 1997, J. Chem. Phys., 106, 3982

Sheehan, C. H. 2000, Ph.D. Thesis, the University of Western Ontario, London, Canada

Smith, M. A., Schlemmer, S., von Richthofen, J., \& Gerlich, D. 2002, ApJ, 578, L87

Teyssier, D., Fossé, D., Gerin, M., et al. 2004, A\&A, 417, 135

Teyssier, D., Hily-Blant, P., Gerin, M., et al. 2005, in Proceedings of the dusty and molecular universe: a prelude to Herschel and ALMA, ed. A. Wilson, ESA SP-577 (Noordwijk, Netherlands: ESA Publications Division), 423

Timmermann, R., Bertoldi, F., Wright, C. M., et al. 1996, A\&A, 315, L281

van der Tak, Floris, F. S. 2006, Royal Soc. London Trans. Ser. A, 364, 1848, 3101

van Dishoeck, E. F. 1988, Rate Coefficients in Astrochemistry, ed. T. J. Millar, \& D. A. Williams (Dordrecht, Boston: Kluwer Academic Publishers), 49

Wakelam, V., \& Herbst, E. 2008, ApJ, 680, 371

Ward-Thompson, D., Nutter, D., Bontemps, S., Whitworth, A., \& Attwood, R. 2006, MNRAS, 369, 1201

Williams, J. P., Bergin, E. A., Caselli, P., Myers, P. C., \& Plume, R. 1998, ApJ, 503,689

Wolfire, M. G., Hollenbach, D., McKee, C. F., Tielens, A. G. G. M., \& Bakes, E. L. O. 1995, ApJ, 443, 152

Wolfire, M. G., Tielens, A. G. G. M., Hollenbach, D., \& Kaufman, M. J. 2008, ApJ, 680, 384

Woodall, J., Agúndez, M., Markwick-Kemper, A. J., \& Millar, T. J. 2006, A\&A, 466, 1197

Woods, R. C., Gudeman, C. S., Dickman, R. L., et al. 1983, ApJ, 270, 583

Wootten, A., Loren, R. B., \& Snell, R. L. 1982, ApJ, 255, 160

Zhou, S., Jaffe, D. T., Howe, J. E., et al. 1993, ApJ, 419, 190

Ziurys, L. M., \& Apponi, A. J. 1995, J, 455, 73 